\title{
The occurrence of the Hall instability in crusts of isolated neutron stars
}

\author{
M. Rheinhardt ${ }^{1}$, D. Konenkov ${ }^{2}$, and U. Geppert ${ }^{1}$ \\ 1 Astrophysikalisches Institut Potsdam, An der Sternwarte 16, 14482 Potsdam, Germany \\ e-mail: urme@aip.de \\ 2 Ioffe Physico-Technical Institute, 194021 Af. F., Politechnicheskaya 26, St. Petersburg, Russia \\ e-mail: dyk@astro.ioffe.rssi.ru
}

Received 15 July 2003 / Accepted 2 March 2004

\begin{abstract}
In former papers we showed that during the decay of a neutron star's magnetic field under the influence of the Hall drift, an unstable growth of small-scale field structures at the expense of the large-scale background field may happen. This linear stability analysis was based on the assumption of a uniform density throughout the neutron star crust, whereas in reality the density and all transport coefficients vary by many orders of magnitude. Here, we extend the investigation of the Hall drift induced instability by considering realistic profiles of density and chemical composition, as well as background fields with more justified radial profiles. Two neutron star models are considered differing primarily in the assumption on the core matter equation of state. For their cooling history and radial profiles of density and composition we use known results to infer the conductivity profiles. These were fed into linear calculations of a dipolar field decay starting from various initial configurations. At different stages of the decay, snapshots of the magnetic fields at the equator were taken to yield background field profiles for the stability analysis. Its main result is that the Hall instability may really occur in neutron star crusts. Characteristic growth times are in the order of $\$ 10^{4} \ldots 10^{6}$ yrs depending on cooling age and background field strength. The influence of the equation of state and of the initial field configuration is discussed.
\end{abstract}

Key words. stars: neutron - stars: magnetic fields

\section{Introduction}

Neutron stars (NSs) are carriers of the strongest magnetic fields which occur in nature. But, astonishingly enough, most of the quantitative studies of magnetic field decay in NS crusts consider only the linear induction equation, i.e., a field decay caused solely by Ohmic dissipation (see, e.g., Urpin et al. 1994; Urpin \& Konenkov 1997; Page et al. 2000).

Yakovlev \& Shalybkov (1991) showed that in a twocomponent plasma the resistivity components parallel and perpendicular to the magnetic field coincide, as a result of which in turn the ambipolar drift disappears. This result is applicable to the electrons in the fully ionized crystal matter of the NS crust, too (see Urpin \& Shalybkov 1999). Further, convective motions will not exist since the crust is almost completely crystallized after, say, $\lesssim 10^{4}$ yrs. Therefore, the magnetic field evolution in the crust is solely determined by Ohmic diffusion and the so-called Hall drift where the latter is the only non-linearity in this process (if the weak and therefore weakly nonlinear back-reaction of the magnetic field on the conductivity via Joule heating is discounted). Recently, two of us (Rheinhardt \& Geppert 2002; Geppert \& Rheinhardt 2002)

Send offprint requests to: M. Rheinhardt, e-mail: mreinhardt@aip.de showed that for a (large-scale) magnetic background field characterized by a sufficiently curved radial profile beyond a certain marginal field strength, the Hall drift may cause an unstable growth of small-scale perturbations. For a homogeneous medium these are concentrated towards the boundary adjacent to vacuum.

We performed this stability analysis based on the linearized induction equation with Hall drift for a homogeneous plane slab of finite thickness, infinitely extended in both horizontal dimensions and bound by vacuum and a medium of infinite conductivity at its upper and lower sides, respectively. While this model is perhaps an acceptable approximation of the NS crust with respect to geometry, the assumption of a spatially uniform density and chemical composition, which results in a uniform scalar conductivity and Hall drift coefficient, is surely a very crude one. Actually, the density and thus the coefficients depending on it may vary throughout the crust by many orders of magnitude (see, e.g., Page et al. 2000). Less pronouncedly, the chemical composition (that is, mass number $A$ and atomic number $Z$ ) varies, too. Additionally, the scalar conductivity and the Hall drift coefficient are dependent on the temperature and the impurity concentration.

The effect of the Hall drift on the magnetic field evolution in NSs has been considered by a number of authors 
(Goldreich \& Reisenegger 1992; Vainshtein et al. 2000; Urpin \& Shalybkov 1995, 1999; Shalybkov \& Urpin 1995, 1997; Naito \& Kojima 1994; Haensel et al. 1990; Hollerbach \& Rüdiger 2002). The only study, however, which includes quantitatively the effect of the crustal density stratification in this context is the one by Vainshtein et al. (2000). They showed that due to a density gradient, the Hall drift may create current sheets, where the field can be dissipated very efficiently. However, the occurrence of the mentioned Hall instability could not be detected by them because in their linear analysis they considered a uniform background field only, i.e., a field which will not become unstable regardless which strength it has. In considering the non-linear evolution of the toroidal field they neglected the coupling with the poloidal one, thus making an instability impossible either, see Eq. (9) below.

Observational evidence for a decay of the large-scale (dipolar) magnetic field of middle aged pulsars, being drastically accelerated in comparison with the purely Ohmic decay, has been discussed in Geppert \& Rheinhardt (2002). Our reasoning there is based on the detection of braking indices greater than three by Johnston \& Galloway (1999), who used measurements of the rotational period $P$, and its temporal derivative $\dot{P}$, dating from different observational epochs. As a typical result we found that the decay times inferred from $P$ and $\dot{P}$ may be smaller than $10^{-4}$ times the (estimated) Ohmic decay time. A possible explanation for such a rapid field decay may be the Hall instability which drains magnetic energy out of the dipolar field and uses it for the build up of small-scale magnetic field structures, which eventually decay by Ohmic dissipation.

The occurrence of small-scale magnetic field structures in the crustal layers of NSs may cause further effects which are potentially observable. Beyond the decrease of the braking power of the magnetic field, the increased small-scale Lorentz forces can trigger a cracking of the crystallized crust. Also, due to a rapid and spatially concentrated Ohmic decay of the smallscale field structures, hot spots may appear in the surface layers. Recent observations, both in the X-ray (Becker et al. 2003), and in the radio range (Gil et al. 2002), support the idea of the existence of strong small-scale magnetic field structures at the NS surface.

Of course, none of the mentioned phenomena can be unambiguously attributed to the effect of the Hall instability. Some possible alternative explanations are discussed in Geppert \& Rheinhardt (2002).

However, the simultaneous existence of a sufficient curvature of the background field profile and of a sufficiently large magnetization parameter $\omega_{B} \tau_{\mathrm{e}}$ related to that field (where $\omega_{B} \tau_{\mathrm{e}}>1$ is a necessary condition) will very likely lead to the appearance of the Hall instability. How vigorously it develops for a realistically modeled crust and at which strength it saturates, are open questions. Also unanswered is, how far it may be hidden by other effects of the field evolution as, e.g., the "normal" Hall cascade. The first of these questions will be addressed here.

One of the most convincing indicators for the importance of the Hall drift in the crust is the evolution of the magnetization parameter $\omega_{B} \tau_{\mathrm{e}}$ there. In Fig. 1 of Geppert \& Rheinhardt (2002) this quantity is shown for different temperatures, that is, for

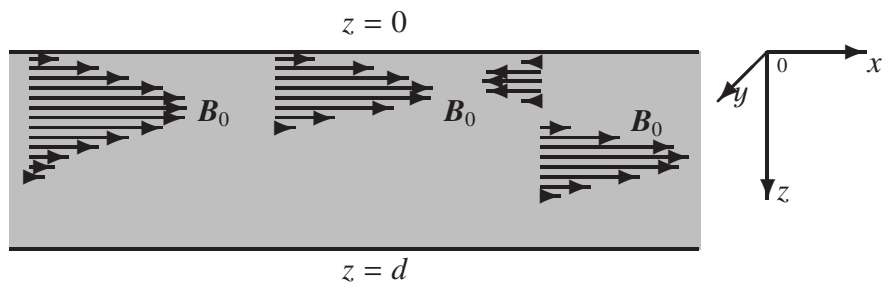

Fig. 1. Sketch of the model geometry ( $\boldsymbol{B}_{0}-$ background fields). In $z>$ $d$ a perfect conductor, in $z<0$ vacuum is assumed.

different ages as a function of density. These results are based on linear field decay calculations for a standard NS model with a medium equation of state (EOS) as presented by Page et al. (2000). Clearly, as soon as the magnetic field strength exceeds $10^{12} \mathrm{G}, \omega_{B} \tau>1$ in some regions of the crust, and the Hall drift begins to dominate the Ohmic decay.

In order to answer the question whether at all and how intensive the Hall instability occurs in real NS crusts, we will consider crustal density profiles resulting from NS models based on stiff and medium EOSs of the core matter. As background magnetic fields which must - in comparison with the unstable field perturbations - evolve very slowly, we use those, calculated by the methods described in Page et al. (2000). The evolution of those large-scale fields is affected only by the density profile of the NS crust and its cooling history, both determined essentially by the EOS. Furthermore, the crustal field evolution is affected by the chemical composition and impurity concentration within the crust, as well as by the initial strength and structure of the field which, in turn, reflect the processes at birth of the NS. These results are insofar incomplete, as they just do not take into account the very efficient drain of magnetic energy from the background field during the period of the Hall instability. They should, however, provide hints under which conditions, more realistic than those considered up to now, an episode of strongly non-linear magnetic field decay may take place.

This paper follows the lines of thought as presented by Rheinhardt \& Geppert (2002), and is organized as follows: in the next section the basic equations of the model are derived, and the assumed properties of the crustal matter are described together with the background field profiles we used. Section 3 briefly outlines the method of solving the eigenvalue problem and provides and discusses the numerical results. Conclusions are drawn in Sect. 4 with a focus on possible observational consequences of the Hall instability.

\section{Description of the model}

\subsection{Basic equations, geometry and boundary conditions}

In the absence of convective motions and of ambipolar diffusion, conditions found in the solid crust of NSs, the equations governing the magnetic field evolution read

$\dot{\boldsymbol{B}}=-c \operatorname{curl}\left(\frac{c}{4 \pi \sigma_{0}}\left(\operatorname{curl} \boldsymbol{B}+\omega_{B} \tau_{\mathrm{e}}\left(\operatorname{curl} \boldsymbol{B} \times \boldsymbol{e}_{B}\right)\right)\right)$

$\operatorname{div} \boldsymbol{B}=0$, 
where $\tau_{\mathrm{e}}$ is the relaxation time of the electrons and $\omega_{B}=$ $e|\boldsymbol{B}| / m_{\mathrm{e}}^{*} c$ the electron Larmor frequency, with $e$ being the elementary charge, $m_{\mathrm{e}}^{*}$ the effective mass of an electron, and $c$ the speed of light. $\boldsymbol{e}_{B}$ is the unit vector in $\boldsymbol{B}$-direction. The scalar conductivity $\sigma_{0}$ is given by

$\sigma_{0}=\frac{Z}{A} \frac{e^{2} \rho\left(1-X_{\mathrm{n}}\right)}{m_{u} m_{\mathrm{e}}^{*}} \tau_{\mathrm{e}}$.

Here $\rho$ denotes the (depth depending) density of the crustal matter, and $m_{u}$ the atomic mass unit. By virtue of their direct dependences on density, both $m_{\mathrm{e}}^{*}$ and $\tau_{\mathrm{e}}$ depend on the crustal depth, too. The mass and atomic numbers $A$ and $Z$, respectively, also depend on depth as well as the fraction of free neutrons $X_{\mathrm{n}}$ (see, e.g., Negele \& Vautherin 1973; Haensel \& Zdunik 1990). Equation (1) lets one expect that the Hall drift described by the term $\omega_{B} \tau_{\mathrm{e}}\left(\operatorname{curl} \boldsymbol{B} \times \boldsymbol{e}_{B}\right)$ may become important for the magnetic field decay only if $\omega_{B} \tau_{\mathrm{e}} \gtrsim 1$.

Linearization of Eq. (1) with respect to a background field (= reference state) $\boldsymbol{B}_{0}$ yields:

$\dot{b}=-\operatorname{curl}(\eta \operatorname{curl} \boldsymbol{b})-\operatorname{curl}\left(\alpha\left(\operatorname{curl} \boldsymbol{B}_{0} \times \boldsymbol{b}+\operatorname{curl} \boldsymbol{b} \times \boldsymbol{B}_{0}\right)\right)$

$\operatorname{div} \boldsymbol{b}=0$

describing the behavior of small perturbations $\boldsymbol{b}$ of the reference state. Here, the magnetic diffusivity $\eta$, and the Hall coefficient $\alpha$ are given by

$\eta=\frac{c^{2}}{4 \pi \sigma_{0}}, \quad \alpha=\eta \frac{\omega_{B} \tau_{\mathrm{e}}}{|\boldsymbol{B}|}=\frac{A}{Z} \frac{m_{u} c}{4 \pi e \rho\left(1-X_{\mathrm{n}}\right)}$.

Note, that the Hall parameter $\alpha$ is time-independent for a nonaccreting NS while $\eta$ depends strongly on the crustal temperature via $\tau_{\mathrm{e}}$ and, therefore, on time. In general, $\boldsymbol{B}_{0}$ is a decaying field, i.e., time-dependent, too. For the instability analysis we nevertheless want to treat them as constants. Then the following necessary condition has to be satisfied to take the results for valid: the growth time of an unstable mode has to be significantly shorter than all the characteristic times of changes of coefficients in Eq. (3), especially significantly shorter than the decay time of the background field. In the following this restriction will be referred to as the "background dynamics permissibility condition".

Employing the arguments from dynamo theory quoted in Rheinhardt \& Geppert (2002), one can conclude that $\alpha \operatorname{curl} \boldsymbol{B}_{0}$ must not be a homogeneous field. More precisely, when interpreting this term as a velocity field, it must not be a rigid body motion, in order to be capable of delivering energy to $\boldsymbol{b}$. Hence, in contrast to the homogeneous density model, now almost any inhomogeneous $\boldsymbol{B}_{0}$ may potentially enable the instability.

Let us now specify the geometry of our model. Idealizing the spherical shell of the NS crust, we consider a plane slab which is infinitely extended both into the $x$ - and $y$-directions, but has a finite thickness $d$ in $z$-direction. We identify $z$ with the crustal depth being zero at the surface of the NS. The background field is assumed to be parallel to the surfaces of the slab pointing, say, in $x$-direction and to be dependent on the depth $z$ only, i.e.,

$\boldsymbol{B}_{0}=B_{0} \boldsymbol{e}_{x}=f(z) \boldsymbol{e}_{x}$, where in comparison to the homogeneous density model the minimum demand on $f(z)$ can now be relaxed from quadratic to linear in $z$, if only $\alpha$ is not a constant (see above). Note, that due to the assumption Eq. (5) $\alpha \operatorname{curl} \boldsymbol{B}_{0} \times \boldsymbol{B}_{0}$ represents a gradient. Thus the unperturbed evolution of the background field is not at all affected by the Hall drift; in the absence of any electromotive force it would decay purely ohmically.

The choice Eq. (5) is not only motivated by the sake of simplicity, but to some extent justified by the physical conditions during the proto-NS stage (after the end of a possible fieldgenerating phase): as long as the layers which later on form the crust are liquid, the magnetic field adjusts to be close to a magnetostatic equilibrium. Then it approximately obeys the condition

$\operatorname{curl}\left(\frac{1}{n_{\mathrm{e}}} \operatorname{curl} \boldsymbol{B} \times \boldsymbol{B}\right)=\mathbf{0}$

with $n_{\mathrm{e}}$ being the electron number density (see Thompson $\&$ Duncan 1993, Sect. 14.1) ${ }^{1}$. As the Hall coefficient $\alpha$ can be written in the form $\alpha=c / 4 \pi n_{\mathrm{e}} e$ (see, e.g., Goldreich \& Reisenegger 1992) this is at the same time the condition for the Hall e.m.f. in Eq. (1) to be a gradient and thus ineffective. However, as the modes of free decay in the crystallized crust are in general violating Eq. (6), the crustal field will tend to deviate increasingly from the magnetostatic configuration. But at least for early stages, one may suppose that although $\omega_{B} \tau_{\mathrm{e}}$ might already be bigger than unity, the effect of the Hall term on the background field is dominated by ohmic dissipation.

In Fig. 1, the model geometry is shown with three different depth profiles of $\boldsymbol{B}_{0}$, depicting qualitatively some of those we employed. They will be specified later.

We decompose a perturbation $\boldsymbol{b}$ into poloidal and toroidal constituents, $\boldsymbol{b}=\boldsymbol{b}_{\mathrm{p}}+\boldsymbol{b}_{\mathrm{t}}$, which can be represented by scalar functions $S$ and $T$, respectively, by virtue of the definitions

$\boldsymbol{b}_{\mathrm{p}}=-\operatorname{curl}\left(\boldsymbol{e}_{z} \times \nabla S\right), \quad \boldsymbol{b}_{\mathrm{t}}=-\boldsymbol{e}_{z} \times \nabla T$,

ensuring $\operatorname{div} \boldsymbol{b}=0$ for arbitrary $S, T$.

For the sake of simplicity, we will confine ourselves to the study of plane wave solutions with respect to the $x$ and $y$-directions, thus making the ansatz

$\left\{\begin{array}{l}S \\ T\end{array}\right\}(\boldsymbol{x}, \tau)=\left\{\begin{array}{l}s \\ t\end{array}\right\}(z) \exp (\mathrm{i} \tilde{\boldsymbol{k}} \tilde{\boldsymbol{x}}+p \tau)$,

where $\tau$ denotes the time, $\tilde{\boldsymbol{k}}=\left(k_{x}, k_{y}\right), \tilde{\boldsymbol{x}}=(x, y)$ and $p$ is a complex time increment.

Inserting Eqs. (7) with (8) into Eq. (3), we finally obtain two coupled ordinary differential equations for the scalars $s$ and $t$ :

$$
\begin{aligned}
& p t-\eta\left(t^{\prime \prime}-\tilde{k}^{2} t\right)-\eta^{\prime} t^{\prime}+\mathrm{i} \alpha k_{x}\left(f^{\prime \prime} s+\tilde{k}^{2} f s-f s^{\prime \prime}\right) \\
&+\mathrm{i} \alpha^{\prime}\left(k_{y} f t+k_{x} f^{\prime} s\right)=0 \\
& p s-\eta\left(s^{\prime \prime}-\tilde{k}^{2} s\right)+\mathrm{i} \alpha\left(k_{x} f t-k_{y} f^{\prime} s\right)=0,
\end{aligned}
$$

1 There exist even dipolar fields in spherical shells satisfying Eq. (6) with $n_{\mathrm{e}}=n_{\mathrm{e}}(r)$ exactly: $\boldsymbol{B}=-\operatorname{curl}(\boldsymbol{r} \times \nabla S)$ with $S=\left(C_{0}\left(r \int r n_{\mathrm{e}} \mathrm{d} r-\right.\right.$ $\left.\left.r^{-2} \int r^{4} n_{\mathrm{e}} \mathrm{d} r\right)+C_{1} r+C_{2} r^{-2}\right) \cos \vartheta ; r, \vartheta-$ spherical co-ordinates. 
where the dash denotes the derivative with respect to $z$, and $\tilde{k}^{2}=$ $k_{x}^{2}+k_{y}^{2}$. In comparison with the corresponding Eqs. (6) in Rheinhardt \& Geppert (2002), the terms $\propto \eta^{\prime}$ and $\alpha^{\prime}$ occur additionally, but they don't affect the energetic conclusions relevant for the existence of the instability.

When completed with appropriate boundary conditions, these equations define an eigenvalue problem with respect to the time increment $p$. The boundary conditions chosen here are transition to vacuum at $z=0$, and to a perfect conductor at $z=d$, respectively, mimicking the superconducting core below the bottom of the crust and an atmosphere with low conductivity above its surface. They read

$$
\begin{array}{ll}
s^{\prime}+\tilde{k} s=t=0 & \text { for } \quad z=0 \\
s=\eta t^{\prime}-\mathrm{i} k_{y} \alpha f t=0 & \text { for } \quad z=d .
\end{array}
$$

These conditions are equivalent to the requirements that all components of the magnetic field are continuous across the vacuum boundary, and that neither the normal component of the magnetic field nor the tangential components of the electric field penetrate the core. For details see Geppert \& Rheinhardt (2002).

The signs of the wavenumbers are irrelevant for the eigenvalues of Eqs. (9) with (10), since the transformations

$k_{x} \rightarrow-k_{x}, \quad p \rightarrow p, \quad s \rightarrow s, \quad t \rightarrow-t$ and

$k_{y} \rightarrow-k_{y}, \quad p \rightarrow p^{*}, \quad s \rightarrow s^{*}, \quad t \rightarrow-t^{*}$

hold ( $*$ is the complex conjugate). Thus it is sufficient to consider the quadrant $k_{x}, k_{y}>0$ of the $k_{x}-k_{y}$-plane only. On the basis of Eq. (11), it can be easily concluded that changing the sign of $f$ is irrelevant, too, because

$f \rightarrow-f, \quad p \rightarrow p^{*}, \quad s \rightarrow s^{*}, \quad t \rightarrow t^{*}$.

As can be inferred from Eq. (9) using standard energy arguments all solutions for $k_{x}=0$ are damped oscillations, being either purely toroidal or purely poloidal. However, for $k_{y}=0$ growing solutions with their poloidal and toroidal parts mutually coupled are well possible if $\mathfrak{J}\left\{k_{x} \int_{0}^{d}(\alpha f / \eta)\left(s^{*} t\right) \mathrm{d} z\right\}>0$.

\subsection{Crustal matter properties}

The main goal of the present paper is to enhance our knowledge on the conditions under which the Hall instability occurs. As one step in this direction we consider a stratified crust characterized by a realistic density profile instead of a homogeneous crust.

The transport coefficients $(\eta, \alpha)$ are strongly dependent on density and chemical composition of the crust (see Eqs. (2) and (4)). Additionally, as far as collisions with phonons are concerned, $\eta$ depends (via $\tau_{\mathrm{e}}$ ) on the crustal temperature, see Urpin \& Yakovley (1980). In turn, the quantities $\rho, A, Z$ and $T$ are in their temporal behavior mainly determined by the EOS of the NS matter, especially that of the core. Therefore, one has first to make a choice among the multitude of proposed core EOSs (see, e.g., van Riper 1991). We decided to consider two of them, which are generally accepted to be typical representatives of a medium (Friedman \& Pandharipande 1981, henceforth FP)
Table 1. Properties of the Pandharipande-Smith (PS) and FriedmanPandharipande (FP) NS models; $M_{\mathrm{NS}}=1.4 M_{\odot}$.

\begin{tabular}{lcc}
\hline \hline & FP & PS \\
\hline EOS & medium & stiff \\
Compactness $G M / c^{2} R$ & 0.2 & 0.13 \\
Central density $\left(\mathrm{g} \mathrm{cm}^{-3}\right)$ & $1.17 \times 10^{15}$ & $3.64 \times 10^{14}$ \\
NS radius $(\mathrm{km})$ & 10.64 & 16.4 \\
Crust thickness (m) & 730 & 3860 \\
& Friedman \& & Pandharipande \\
Reference & Pandharipande & \& Smith \\
& $(1981)$ & $(1975)$ \\
\hline
\end{tabular}
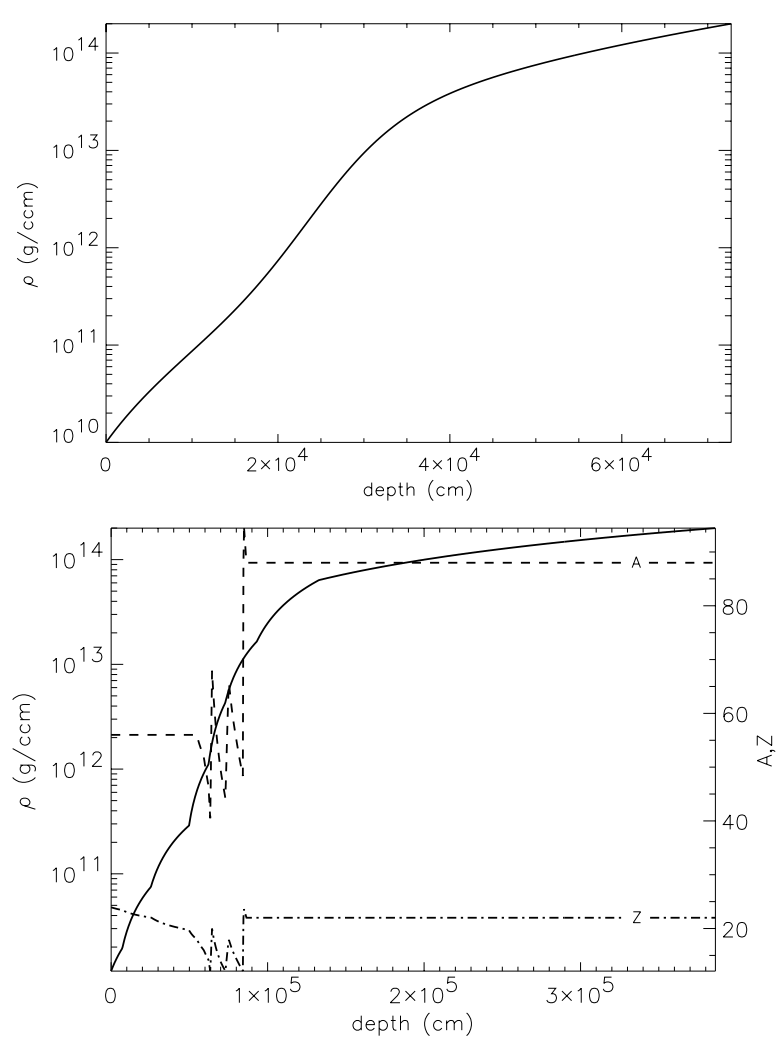

Fig. 2. Radial density (solid) profiles for the FP (top) and PS (bottom) models. Additionally, for the latter the chemical composition, $A, Z$, (dashed and dash-dotted, respectively) is given.

and a stiff (Pandharipande \& Smith 1975, henceforth PS) EOS, respectively, thus probably covering an essential part of the observed NSs. (The extreme soft EOSs, e.g., that derived by Baym et al. (1971), are now considered unlikely to be realized in nature, see Page et al. 2000) Further on, we specify the crust in the FP case to consist of cold catalyzed matter, and in the PS case to be dominated by matter accreted and processed in the past. The corresponding EOSs of the crustal matter and its chemical compositions were derived in Negele \& Vautherin (1973) and Haensel \& Zdunik (1990), respectively. Since these two models are referred to and utilized in numerous papers, we thus hope to facilitate discussion and application of our results. 


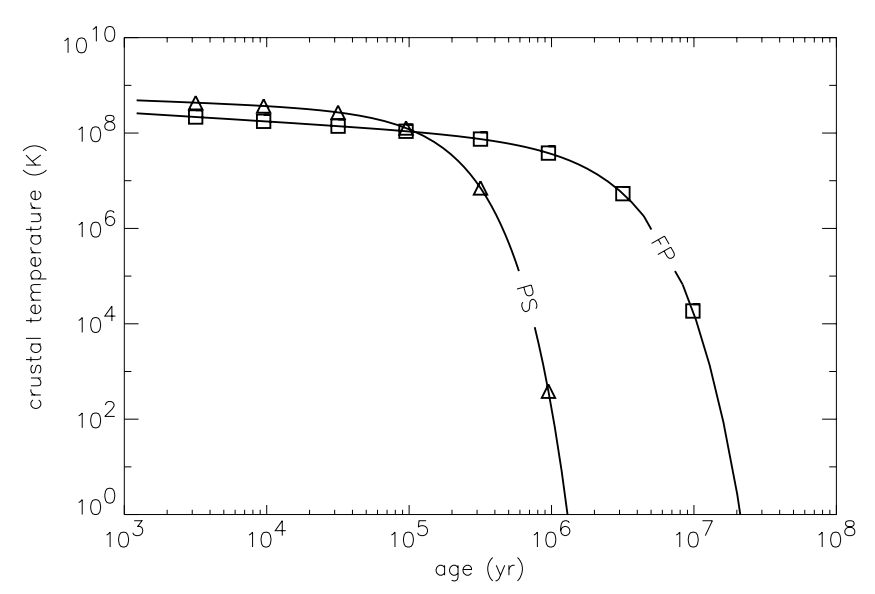

Fig. 3. Cooling curves for the FP (squares) and PS (triangles) models. The symbols denote the ages at which the stability analysis is performed.

Some characteristic properties of the selected models resulting from their density structure specified for $M_{\mathrm{NS}}=1.4 M_{\odot}$, are summarized in Table 1; Fig. 2 shows the density profiles. Additionally, the profiles of the chemical composition, that is, $A$ and $Z$, for the PS model as derived from the data in Haensel \& Zdunik (1990) are given. There exist a number of discrete depths (i.e., densities and pressures) where the preferred species of nuclei change almost abruptly causing discontinuities in $A$ and $Z$. For the FP model, the corresponding data were given only implicitly in the form of a smooth fitting function for $\rho(z)$. In both cases the crust-core boundary was assumed to be at a density of $2 \times 10^{14} \mathrm{~g} \mathrm{~cm}^{-3}$ whereas the crust surface was defined by the density $10^{10} \mathrm{~g} \mathrm{~cm}^{-3}$.

The most important of the EOS's direct consequences is the degree of compactness: the smaller compactness (i.e., larger radius and crust thickness) of the PS model compared with the FP model of equal mass, results from its stiffer EOS. Correspondingly, the FP model cools down significantly slower than the former for ages higher than $10^{5} \mathrm{yrs}$, since a large compactness (strong gravity) inhibits the heat transfer (see, e.g., Misner et al. 1973). Figure 3 shows the cooling curves for both models as calculated by van Riper (1991). Throughout this paper we confine ourselves to NSs old enough (say, some $10^{3} \mathrm{yrs}$ ) that the crustal temperature can be considered uniform, and the density profile is no longer changing in time.

Finally, Fig. 4 gives the profiles of the magnetic diffusivity $\eta$ and the Hall parameter $\alpha$ for both models. Different profiles of $\eta$ are shown corresponding to the ages at which the stability analysis will be performed, but only one profile for $\alpha$ as it doesn't depend on temperature and hence not on age. For $\eta$ an impurity concentration $Q=0.01$ was assumed $^{2}$. Note, that in contrast to the PS model merely the implicit influence of $A$ and $Z$ on $\eta$ and $\alpha$ via the density is included for

2 This value is perhaps reasonable, but uncertain by at least a factor of 10 either way. If the conductivity is clearly dominated by impurity scattering, that is, for ages $\gtrsim 10^{6} \mathrm{yrs}$, depending on the cooling history, a noticeable influence of $Q$ on the growth times presented below could in principle exist.
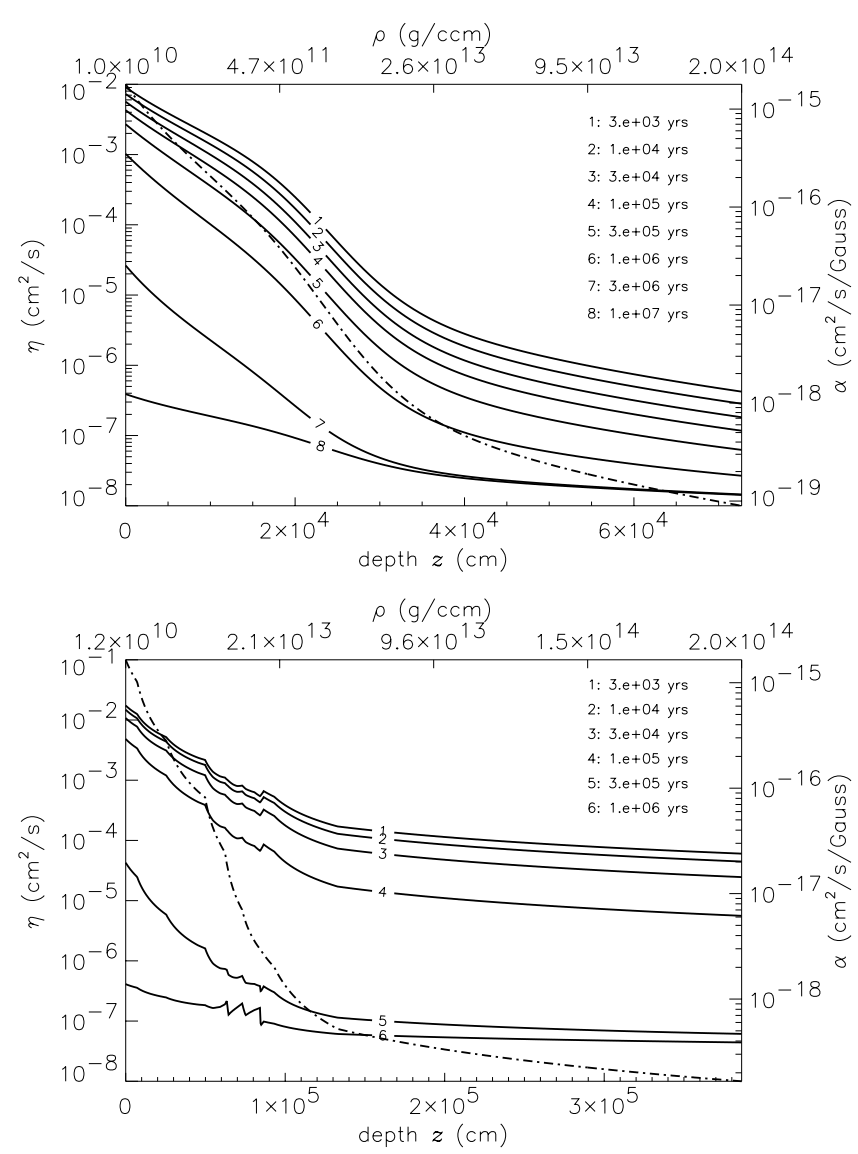

Fig. 4. Radial profiles of diffusivity $\eta$ (solid) for different ages of the NS, and Hall coefficient $\alpha$ (broken). Top: FP, bottom: PS model. All $\eta$ profiles for ages $\geq 10^{7}$ yrs (FP) and $\geq 10^{6}$ yrs (PS) practically coincide.

the FP model, the explicit one being neglected as well as the influence of $X_{\mathrm{n}}$.

Since the cooling of a NS proceeds faster in the PS model, further cooling will not affect the conductivity after about $5 \times$ $10^{5}$ yrs in this case. Then phonons are practically no longer excited in the crustal crystal and the conductivity is determined by electron-impurity collisions alone. Hence, the diffusivity doesn't change any more, while for the FP model a significant number of phonons may be excited up to an age of approximately $5 \times 10^{6} \mathrm{yrs}$; therefore $\eta$ depends on time until this age.

According to the discontinuous behavior of $A$ and $Z$ in the PS model (see Fig. 2), a non-smooth behavior of its transport coefficients appears in Fig. 4. We decided not to smooth out these discontinuities because it had meant introducing unnecessarily some further arbitrariness into the model.

\subsection{Background field}

As the second major ingredient of a more realistic crust model we have to specify background field profiles more justified with a view to NS physics than the ad hoc assumptions employed in our former papers. That's why we turn at this point to a model simulating the Ohmic decay of the magnetic field in a cooling NS's crust (i.e., in a spherical shell), which is just the situation where we expect the most significant effects of the Hall 
instability to occur. When thinking about initial conditions for such simulations, one has to cope with the fact that, unfortunately, there is less certainty about the very origin of the NSs' magnetic fields. Favorite mechanisms of magnetic field generation are proto-NS dynamos (Thompson \& Duncan 1993) and thermoelectric instabilities (see, e.g., Wiebicke \& Geppert 1996), but inheriting the magnetic field from the NS's progenitor seems well possible, too. Since the way of field generation is surely significant with respect to the details of the field structure, there seems to be no other resort when having to specify it than the principle of greatest possible simplicity. Therefore, we decided to consider dipolar fields only. Further on, it seems to be reasonable to assume with respect to their radial profiles that not all parts of the (in the case of a proto-NS dynamo: later) crust equally participate in the field generation. Another process to be taken into account, is the fallback accretion burying the magnetic field again within the crust soon after its emergence.

On the other hand and somewhat conflicting with maximum simplicity, we had to ensure that enough curvature is 'injected' into the radial magnetic field profile initially. See Rheinhardt \& Geppert (2002) for the essential role of the second derivative of the background field's profile (which is partly taken over by the gradient of $\alpha$ in the present case as discussed above).

At this point it is necessary to discuss the relationship between the only $z$-dependent $\boldsymbol{B}_{0}$ profile used in the plane slab geometry, and a both $r$ - and $\theta$-depending profile resulting from decay calculations in a spherical shell. Since $\boldsymbol{B}_{0}$ as assumed in Eq. (5) must be parallel to the boundaries, the plane slab model is only meaningful in the close vicinity of the magnetic equator $\theta=\pi / 2(\theta-$ polar angle $)$. $f(z)$ for a certain instant $\tau$ has therefore to be identified with the $\theta$-component of $\boldsymbol{B}(r, \theta, \tau)$ where $r=R-z, \theta=\pi / 2$. Accordingly, the initial background field is completely specified in geometry and strength after having prescribed $f(z)$ for $\tau=0$, which we denote as $f_{\text {in }}$. (The restriction to background fields parallel to the slab boundaries can be relaxed to fields with a constant vertical component. We will treat this case in a forthcoming paper.)

In view of all these aspects, we generally chose initial profiles showing at least one maximum inside the crust and being zero beneath a certain initial penetration depth $z_{\text {in }}$. We fixed the latter by prescribing the corresponding density $\rho_{\text {in }}$ to be either $10^{13}$ or $10^{12} \mathrm{~g} \mathrm{~cm}^{-3}$. The smaller $\rho_{\text {in }}$ results in larger derivatives of the background field profiles thus possibly favoring the instability at early instances. On the other hand the decay will be accelerated and we have to expect smaller growth rates at higher ages (see Sect. 3). The following initial profiles of the 3rd and higher degrees were chosen:

$$
\begin{array}{ll}
f_{\text {in }}(z)=-2 B_{\mathrm{d}}\left(\frac{R}{z_{\text {in }}}\right)^{2} \frac{z}{R-z}\left(1-\left(\frac{z}{z_{\text {in }}}\right)^{2}\right) & \text { cubic } \\
f_{\text {in }}(z)=-4 B_{\mathrm{d}}\left(\frac{R}{z_{\text {in }}}\right)^{4} \frac{z^{3}}{R^{2}(R-z)}\left(1-\left(\frac{z}{z_{\text {in }}}\right)^{4}\right) & \text { heptic } \\
f_{\text {in }}(z)=\pi B_{\mathrm{d}} \frac{R}{z_{\text {in }}} \frac{R}{R-z} \sin \left(4 \pi \frac{z}{z_{\text {in }}}\right) & \text { sinusoidal. }
\end{array}
$$

Here, $B_{\mathrm{d}}$ is just the initial polar surface magnetic field. Note, that the denominator $R-z$ in Eqs. (13)-(15) doesn't influence the degree of $f_{\text {in }}$ with respect to $z$ significantly since inside the crust $R-z \approx R$.

The decay calculations are straightforward (for details see Page et al. 2000); we only mention that the back-reaction of the magnetic field on the temperature distribution and cooling history via Joule heating was neglected. Hence, the results depend linearly on the initial conditions. The same boundary conditions as referred to for the magnetic field perturbations were used. However, we refrained from adjusting the initial profiles Eqs. (13)-(15) to the vacuum boundary condition at the surface since the decay simulation will do this job automatically. (Likewise, diffusion smoothes out the "kink" at $z=z_{\text {in. }}$.) All runs were performed with $B_{\mathrm{d}}=10^{13} \mathrm{G}$ and snapshots of the decaying field were taken at nine instants from $3 \times 10^{3}$ to $3 \times 10^{7}$ yrs in order to define the profiles $f(z)$. Additionally, we scaled them (that is, $B_{\mathrm{d}}$ ) by factors 2 or 5 to study the dependence of the instability on the initial field strength.

Figures 5 to 7 show typical examples of the resulting $f(z)$ for both models. (See also Fig. 1 for schematic sketches of characteristic background fields.) Moreover, these figures present the magnetization parameter $\omega_{B_{0}} \tau_{\mathrm{e}}$ and - only for the FP model, in Fig. 5 - the quantity $\left(\alpha f^{\prime}\right)^{\prime} / \eta$, further on referred to as "curvature parameter". It is an estimate for the ratio of the potentially energy-delivering term $\operatorname{curl}\left(\alpha \operatorname{curl} B_{0} \times \boldsymbol{b}\right)$ to the (anyway) energy-dissipating term $\operatorname{curl}(\eta \operatorname{curl} b)$ in Eq. (3). (Note that the term curl $\left(\alpha \operatorname{curl} b \times B_{0}\right)$ is energetically neutral.) Therefore we suppose that the curvature parameter rather than $\omega_{B_{0}} \tau_{\mathrm{e}}$ itself is the key indicator with respect to the vigour of the instability. For the PS model, the curvature parameter is only piecewise continuous due to the non-smooth profiles of $\eta$ which result in $f^{\prime \prime}$ profiles only piecewise continuous, too. Nevertheless, the solutions of Eqs. (9) are well defined, at least in the mathematically weak sense.

On the other hand, we want to stress that the magnitude of the curvature parameter alone is again not sufficient to infer the existence of the instability, which relies on a complicated interplay of vector quantities. Therefore, an additional qualitative criterion must be satisfied in order to have the potentially energy-delivering term in Eq. (3) really energydelivering. From simple qualitative considerations with axisymmetric fields in a homogeneous medium it is possible to conclude that $f^{\prime \prime}(z) f(z)<0$ has to be satisfied, at least over a certain $z$-interval. Or generalized: background field and curvature parameter must have different signs:

$\left(\alpha f^{\prime}\right)^{\prime}(z) f(z)<0$.

\section{Results}

\subsection{Numerics}

The system of ordinary differential Eqs. (9) was discretized by help of symmetric difference formulae of second order; near boundaries asymmetric formulae were employed, if necessary. In most cases an equidistant grid with a typical node number of 1200 was used, but in quite a number of spot checks 

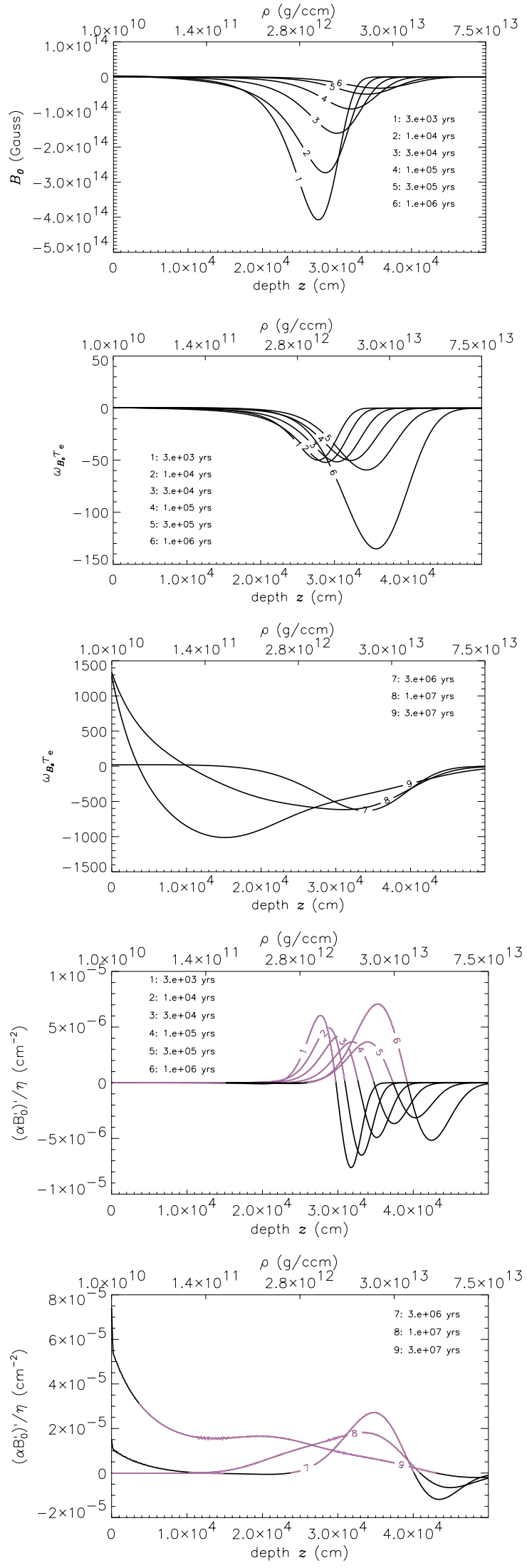

Fig. 5. Background field $B_{0}$, magnetization parameter $\omega_{B_{0}} \tau_{\mathrm{e}}$ and curvature parameter $\left(\alpha B_{0}^{\prime}\right)^{\prime} / \eta$ resulting from the FP model with sinusoidal initial profile Eq. (13) and initial penetration density $\rho_{\text {in }}=10^{13} \mathrm{~g} \mathrm{~cm}^{-3}$, and $B_{\mathrm{d}}=10^{13} \mathrm{G}$. Grey (in color: red) parts indicate adherence to the sign condition Eq. (16).
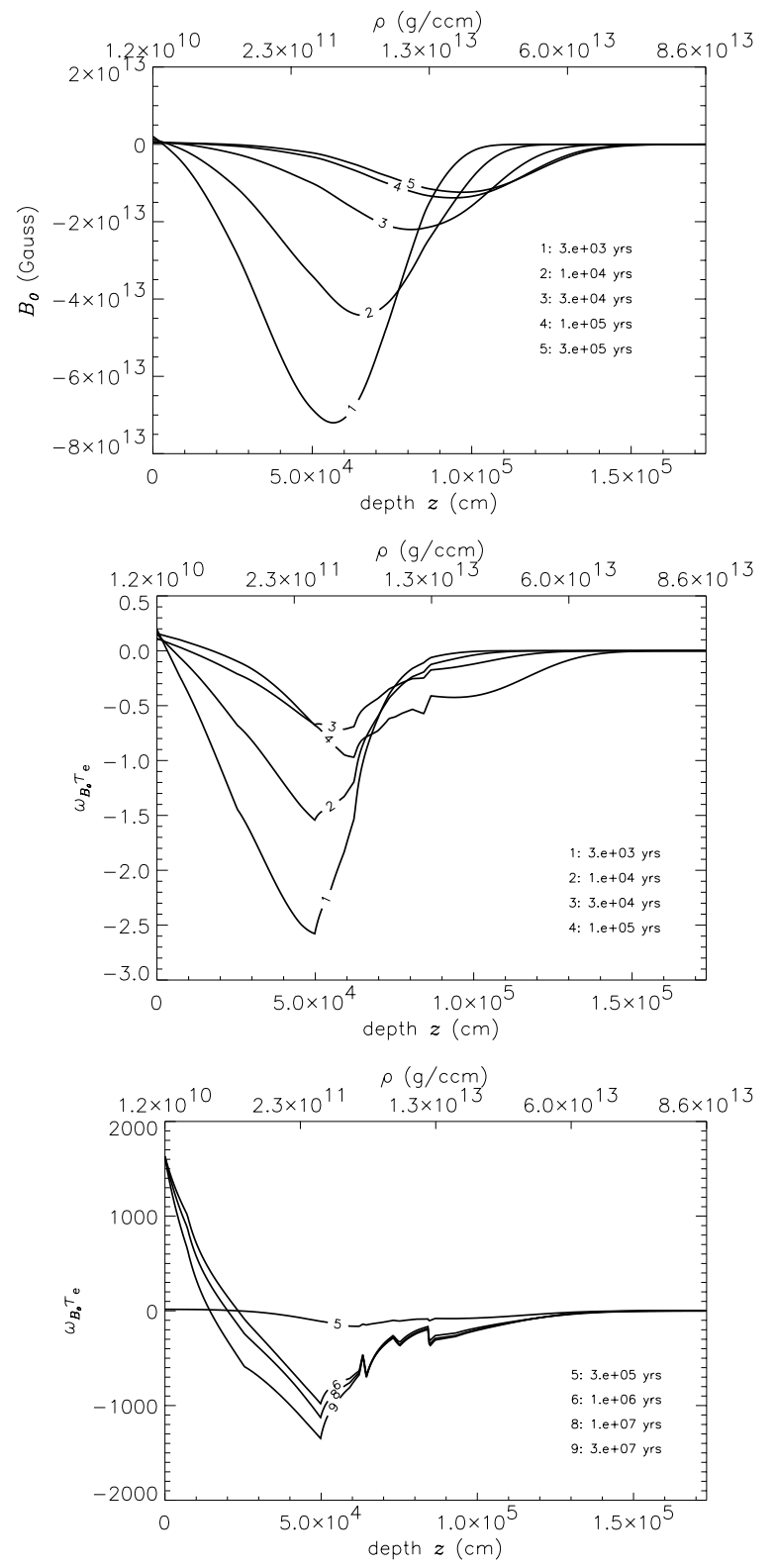

Fig. 6. Background field $B_{0}$ and magnetization parameter $\omega_{B_{0}} \tau_{\mathrm{e}}$ resulting from the PS model with cubic initial profile Eq. (13) and initial penetration density $\rho_{\text {in }}=10^{13} \mathrm{~g} \mathrm{~cm}^{-3}$, and $B_{\mathrm{d}}=10^{13} \mathrm{G}$.

of convergence the node number was repeatedly doubled up to 9600 maximum.

For the numerical solution of the resulting complex nonHermitian standard matrix eigenvalue problem we took benefit of the package ARPACK (routines znaupd, zneupd). Some results were checked applying the LAPACK routine zgeev. The results from both packages agree in at least six digits (but comparisons were possible for moderate node numbers only).

Unfortunately, the order of the difference formulae was not always reflected by the convergence rate of the eigenvalues with respect to the node number: for the PS model convergence was usually significantly slower. Perhaps this behavior is connected with the discontinuities of the coefficients $\eta^{\prime}, \alpha^{\prime}$ and $f^{\prime \prime}$ described above. This supposition is 

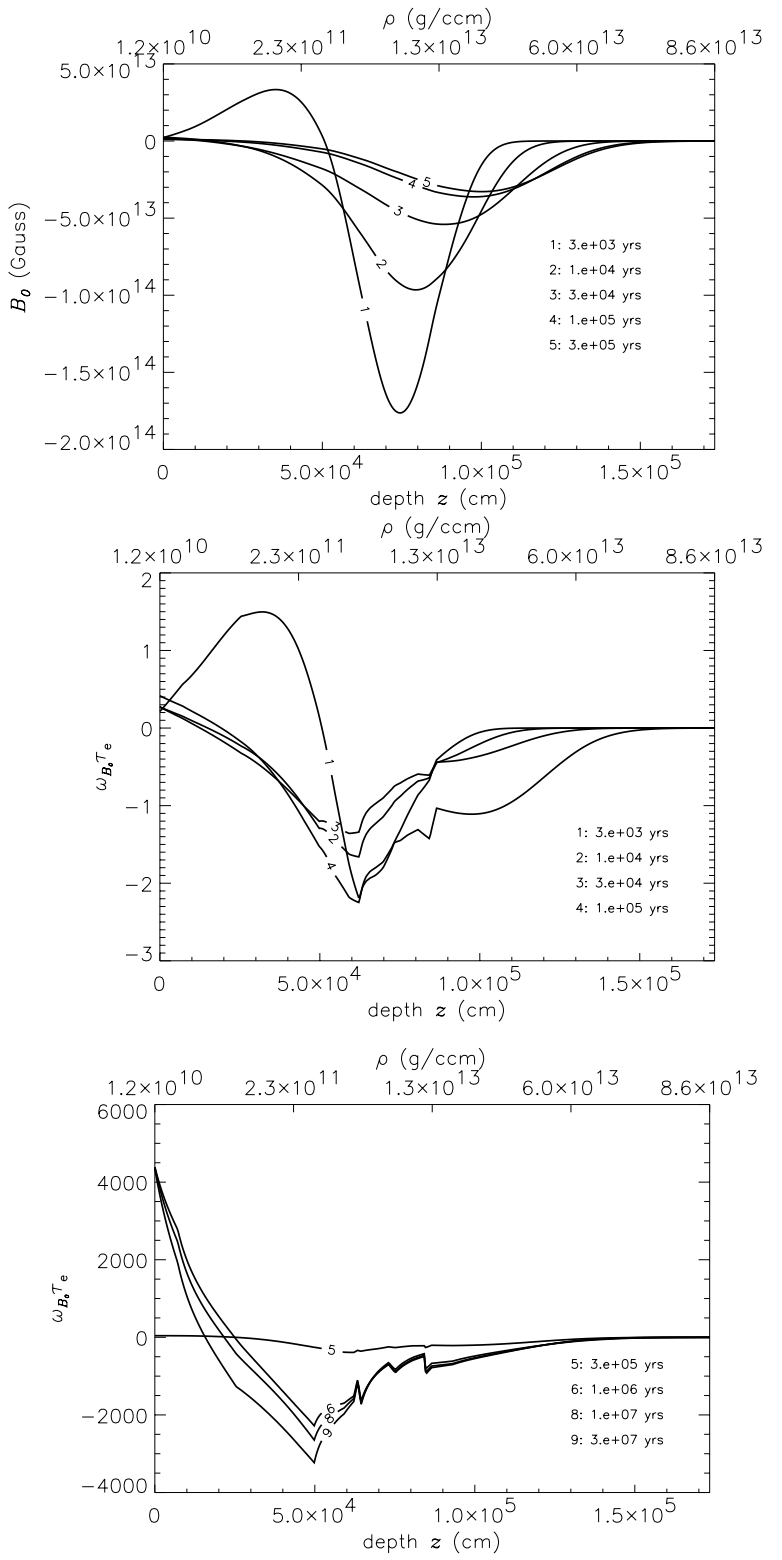

Fig. 7. As Fig. 6, but with sinusoidal initial profile Eq. (15).

supported by the fact that the convergence rates of the FP model are close to (although not exactly) quadratic.

\subsection{General aspects of the results}

As with the homogeneous density model, both oscillating and non-oscillating unstable modes exist, where the fastest growing mode of any specific model turned out to be always amongst the latter. As another common feature of the fastest growing modes, their wavenumber $k_{y}$ has always been found to be zero. When deriving the background field from an axisymmetric poloidal one in the vicinity of its magnetic equator (as we did), association of the $y$ co-ordinate with longitude is surely the proper choice. Thus, with some care one may suppose that in a spherical shell the most unstable modes are preferentially axisymmetric.
Figure 8 shows growth rate $\mathfrak{R}(p)$ and oscillation frequency $\mathfrak{J}(p)$ as functions of $k_{x}$ and $k_{y}$ for a typical example (PS model, age $=3 \times 10^{5} \mathrm{yrs}$, cubic initial field, $B_{\mathrm{d}}=$ $\left.5 \times 10^{13} \mathrm{G}, \rho_{\text {in }}=10^{13} \mathrm{~g} \mathrm{~cm}^{-3}\right)$. The rectangle enclosing the unstable region in the $k_{x}-k_{y}$-plane is defined by $0.4 / d \leq k_{x} \leq$ $18.4 / d, 0 \leq k_{y} \leq 34.4 / d$. But, when obeying the background dynamics permissibility condition (see Sect. 2.1) only a considerably smaller unstable region can be considered feasible.

In contrast to the growth rates, the oscillation frequencies grow in general with $k_{y}$; their highest values seem to occur always at the edge of the unstable region in the $k_{x}-k_{y}$-plane, that is, for $\mathfrak{R}(p) \rightarrow 0$ (and $k_{y} \neq 0$ ). Note, that the oscillating unstable modes can be regarded as helicoidal waves with growing amplitudes. (Cf. Vainshtein et al. (2000) who considered damped helicoidal waves in a stratified crust.)

In Figs. 9 to 14 the growth times of the fastest growing modes $1 / \max (\mathfrak{R}(p)$ ) (simply referred to as "growth time" $\tau_{\text {growth }}$ of a specific model) for the most important cases considered, are presented as functions of age and initial field strength $B_{\mathrm{d}}$. In addition, the tangential period lengths of these modes, $\lambda^{\max }$, are given; because of $k_{y}^{\max }=0$ this quantity is defined as

$\lambda^{\max }=\frac{2 \pi}{k_{x}^{\max }}$.

It should be regarded as one of the dominating scales of the unstable modes since, in contrast to the homogeneous density model, in the majority of cases no prominently small radial scales were found (see Fig. 15). Instead, the radial scales of the unstable modes seem to be determined simply by the radial scale of the background field. Except for some earlier stages of the PS model, $\lambda^{\text {max }}$ depends in general only slightly on $B_{\mathrm{d}}$. Since only discrete equidistant values were assigned to the wavenumber $k_{x}$, the period lengths for different values of $B_{\mathrm{d}}$ frequently even coincide (indicated in the figures by filled symbols instead of open ones).

\subsection{Growth times}

We stress again that the growth times have to be considered as referring to snapshots only. That is, the value for a given NS age has been calculated assuming that the instability starts just at that age, with the background field given at exactly that moment. Clearly, the results must not be interpreted as a sort of temporal evolution of the growth times: any occurrence of the Hall instability will affect the strength and structure of the background field, thereby changing the conditions for the occurrence of the instability itself at later moments. This shortcoming of the results induces us to emphasize once more the necessity of full non-linear calculations.

As an overall property, we state that the dependence of $\tau_{\text {growth }}$ upon the initial polar magnetic field $B_{\mathrm{d}}$ is always monotonously falling.

As long as the Hall term dominates the ohmic one, what is generally true for the most rapidly growing modes, the growth times are insensitive with respect to the diffusivity $\eta$. Therefore, the high uncertainty of the impurity concentration $Q$ (see Sect. 2.2) doesn't affect noticeably the instability. 

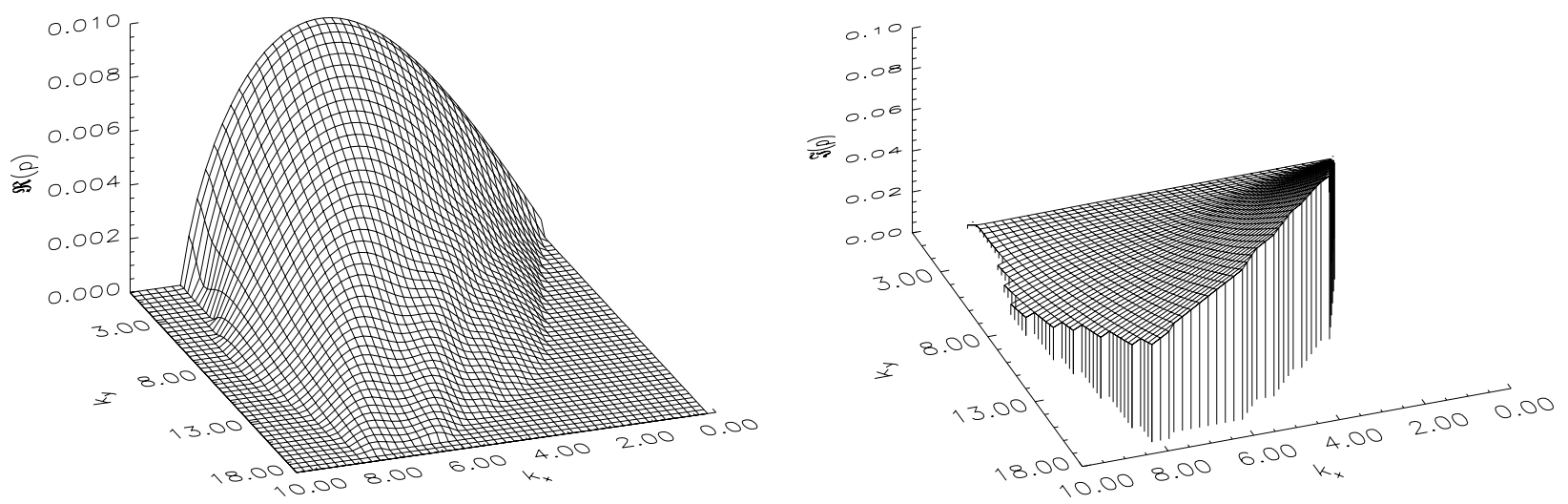

Fig. 8. Eigenvalue $p$ as function of the wave numbers $k_{x}, k_{y}$ for the PS model with cubic initial background field profile Eq. (13), initial field $B_{\mathrm{d}}=5 \times 10^{13} \mathrm{G}$, initial penetration density $\rho_{\text {in }}=10^{13} \mathrm{~g} \mathrm{~cm}^{-3}$, age $3 \times 10^{5}$ yrs. Left: growth rate $\mathfrak{R}(p)$, negative values were set to zero; right: frequency $\mathfrak{J}(p)$, not shown for $\mathfrak{R}(p)<0$. $p$ in units of $4 /(d / \mathrm{cm})^{2} \mathrm{~s}^{-1}=2.68 \times 10^{-11} \mathrm{~s}^{-1}$, wave numbers in units of $2 / d=5.18 \times 10^{-6} \mathrm{~cm}^{-1}$.

Table 2. Growth times $\tau_{\text {growth }}$ (yrs) of the FP model. For $B_{\mathrm{d}}=5 \times 10^{13} \mathrm{G}$ the global minimum and maximum occur always at the ages of $3 \times 10^{3}$ and $3 \times 10^{6}$ yrs, resp. For $B_{\mathrm{d}}=10^{13} \mathrm{G}$ the global minimum occurs always at the age of $3 \times 10^{3}$ yrs. For the cubic profile and $\rho_{\text {in }}=$ $10^{12} \mathrm{~g} \mathrm{~cm}^{-3}$ no unstable modes exist between the ages $10^{4}$ and $3 \times 10^{5} \mathrm{yrs}$.

\begin{tabular}{|c|c|c|c|c|c|c|c|}
\hline \multicolumn{8}{|c|}{$B_{\mathrm{d}}(\mathrm{G})$} \\
\hline & \multirow{2}{*}{$\frac{\text { Profile type }}{\rho_{\text {in }}\left(\mathrm{g} \mathrm{cm}^{-3}\right)}$} & \multicolumn{2}{|c|}{ Cubic } & \multicolumn{2}{|c|}{ Heptic } & \multicolumn{2}{|c|}{ Sinusoidal } \\
\hline & & $10^{13}$ & $10^{12}$ & $10^{13}$ & $10^{12}$ & $10^{13}$ & $10^{12}$ \\
\hline \multirow{3}{*}{$\begin{array}{l}\stackrel{m}{\circ} \\
\stackrel{1}{x} \\
n\end{array}$} & Global minimum & 850 & 1700 & 510 & 750 & 550 & 630 \\
\hline & Global maximum & 256060 & 1150350 & 107650 & 561050 & 53590 & 382800 \\
\hline & Value at highest age & 84880 & 326520 & 38780 & 185630 & 20240 & 122370 \\
\hline \multirow{4}{*}{$\stackrel{\cong}{\circ}$} & Global minimum & 7880 & 74900 & 3470 & 8290 & 2870 & 5620 \\
\hline & Global maximum & 1280780 & 12767800 & 536040 & 3711550 & 266740 & 192520 \\
\hline & occurs at age & $3 \times 10^{6}$ & $10^{6}$ & $3 \times 10^{6}$ & $3 \times 10^{5}$ & $3 \times 10^{6}$ & $3 \times 10^{6}$ \\
\hline & Value at highest age & 358870 & 1466830 & 177270 & 720110 & 88050 & 517920 \\
\hline
\end{tabular}

\subsubsection{FP model}

The growth times of the FP model (Figs. 9 to 11, Table 2) have in common that they start with small values (most in the order of magnitude $10^{3} \mathrm{yrs}$ and smaller) at the youngest age, exhibit a nearly linear dependence on age (a power law with an exponent between 0.8 and 1) until $10^{5} \ldots 3 \times 10^{5} \mathrm{yrs}$, reach a maximum at $10^{6} \ldots 3 \times 10^{6} \mathrm{yrs}$, and fall progressively with age later on. At early stages the background dynamics permissibility condition is well fulfilled for $B_{\mathrm{d}}=5 \times 10^{13} \mathrm{G}$ but must be put in question in most cases with $B_{\mathrm{d}}=10^{13} \mathrm{G}$. Analogously, the maximum growth times are reliable for most cases with $\rho_{\text {in }}=10^{13} \mathrm{~g} \mathrm{~cm}^{-3}$, but questionable for some of the cases with $\rho_{\text {in }}=10^{12} \mathrm{~g} \mathrm{~cm}^{-3}$ and $B_{\mathrm{d}}=10^{13} \mathrm{G}$. At the latest stage considered all the growth times satisfy the condition. We note that the dependence of the growth rate on $B_{\mathrm{d}}$ is not far from being linear for $\rho_{\text {in }}=10^{13} \mathrm{~g} \mathrm{~cm}^{-3}$, whereas for $\rho_{\text {in }}=10^{12} \mathrm{~g} \mathrm{~cm}^{-3}$ the dependence is of higher order. In this case, with the cubic profile and $B_{\mathrm{d}}=10^{13} \mathrm{G}$ even a "gap" in the growth time curve occurs: from the age of $10^{4}$ to the age of $3 \times 10^{5}$ years there were no growing modes to be found at all.

Comparing the growth times for different values of $\rho_{\text {in }}$, one has to state that the smaller initial penetration depth $\left(\rho_{\text {in }}=10^{12} \mathrm{~g} \mathrm{~cm}^{-3}\right)$, although being connected with stronger gradients of the background field, is nevertheless disfavoring the instability. This tendency becomes increasingly apparent with growing age. We explain it with the accelerated decay of these "shallower" profiles.

Comparing the growth times for different initial profile types, one can see that the values for the heptic and the sinusoidal profiles are close together at the earlier stages and clearly smaller than the ones for the cubic profile. However, the late stages reveal the sinusoidal profile to be the favorite; in the case of $\rho_{\text {in }}=10^{13} \mathrm{~g} \mathrm{~cm}^{-3}$ it yields the smallest late stage growth time at all (20240 yrs). As a rule of thumb, the growth times for the cubic, heptic and sinusoidal profiles behave like 4:2:1 at late stages. These ratios reflect even more or less quantitatively the relationship of the corresponding curvature parameters at the late stages, see Fig. 5.

\subsubsection{PS model}

A clearly pronounced and characteristic common property of the data shown in Figs. 12 to 14 and Table 3, is the independence of $\tau_{\text {growth }}$ on age for stages older than $3 \times 10^{5}$ yrs. Further 


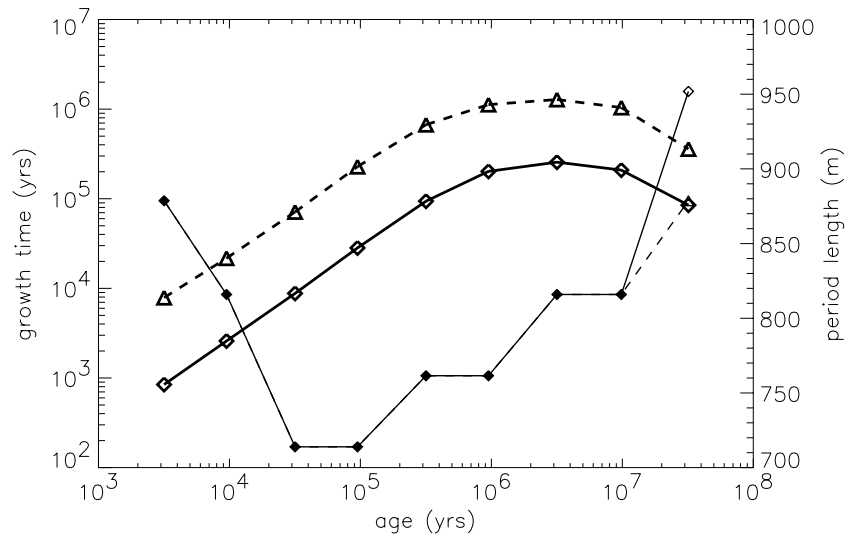

Fig. 9. Growth times and tangential period lengths of the fastest growing modes for the FP model with cubic initial background field profile Eq. (13), initial penetration density $\rho_{\text {in }}=10^{13} \mathrm{~g} \mathrm{~cm}^{-3}$. Solid, diamonds: $B_{\mathrm{d}}=5 \times 10^{13} \mathrm{G}$; dashed, triangles: $B_{\mathrm{d}}=10^{13} \mathrm{G}$. Thick lines, big symbols: growth times; thin lines, small symbols: period lengths. Full small diamonds: coincidence of the period lengths for different values of $B_{\mathrm{d}}$.

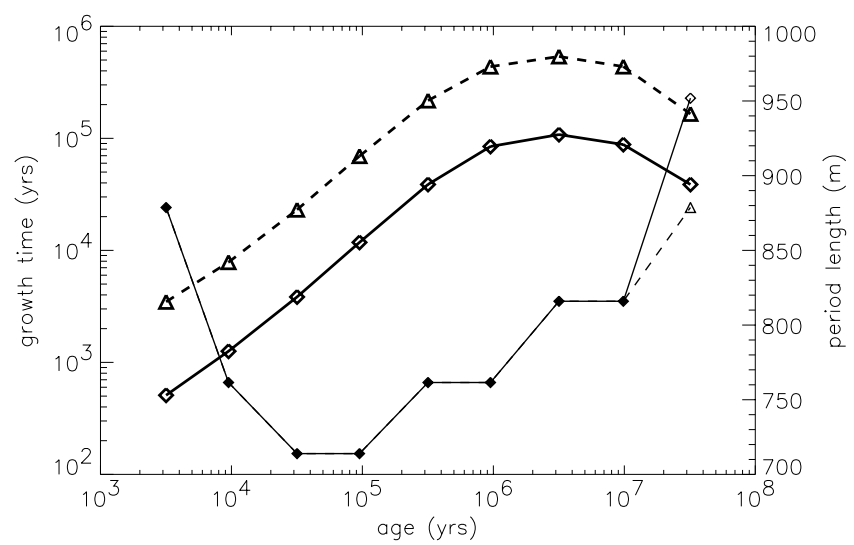

Fig. 10. As Fig. 9, but with heptic initial background field profile Eq. (14).

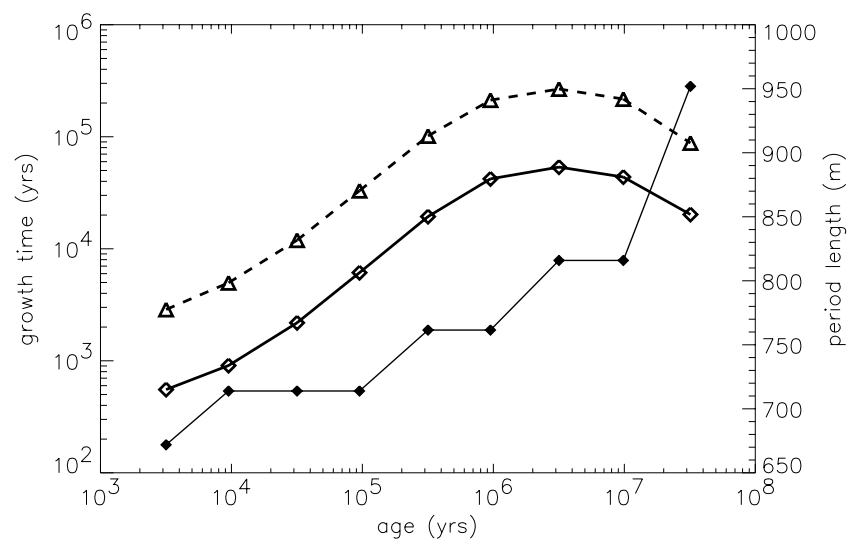

Fig. 11. As Fig. 9, but with sinusoidal initial background field profile Eq. (15).

on, for these ages the dependence of $\tau_{\text {growth }}$ on $1 / B_{\mathrm{d}}$ is linear to a high accuracy. We explain the first effect by referring to the weak dependence of the curvature parameter $\left(\alpha f^{\prime}\right)^{\prime} / \eta$ on age

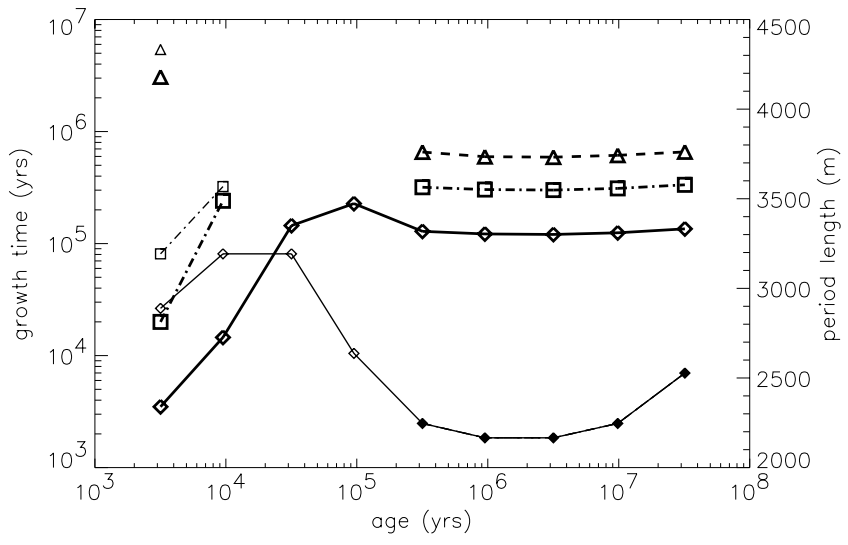

Fig. 12. As Fig. 9, but for the PS model; dash-dotted, squares: $B_{\mathrm{d}}=2 \times 10^{13} \mathrm{G}$.

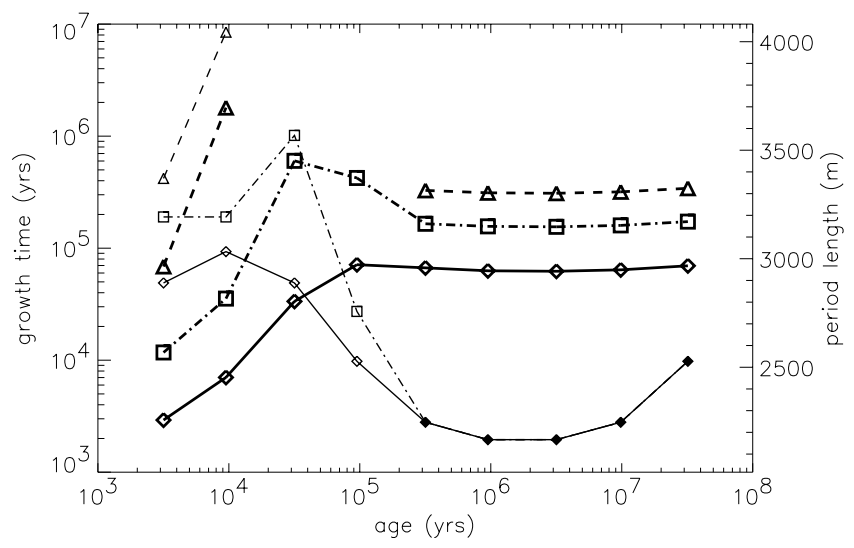

Fig. 13. As Fig. 12, but with heptic initial background field profile Eq. (14).

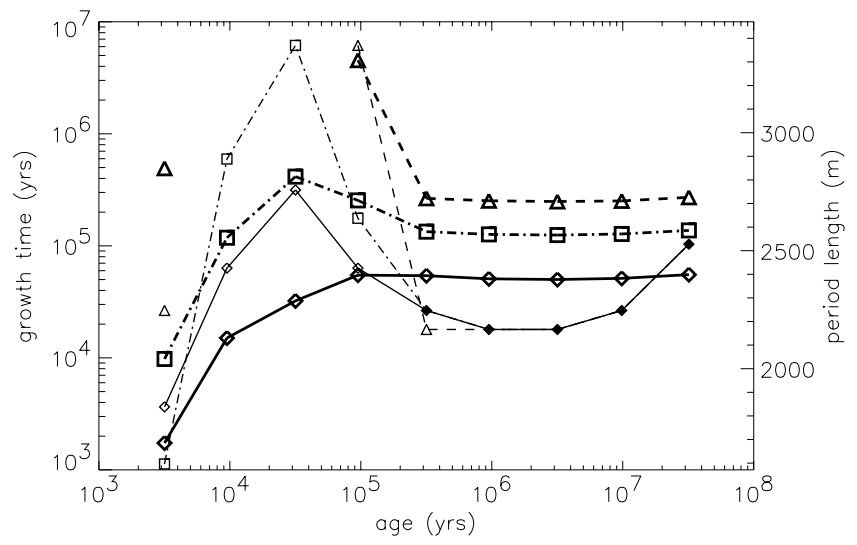

Fig. 14. As Fig. 12, but with sinusoidal initial background field profile Eq. (15).

(see Figs. 6 and 7) and the second by stating the dominance of the Hall terms in Eq. (3) in this range of ages.

In contrast, for the earlier stages the sensitivity of the growth times with respect to $B_{\mathrm{d}}$ is extraordinarily high. It is highest for the cubic profile for which at the age of $3 \times 10^{3} \mathrm{yrs}$ a scaling of $B_{\mathrm{d}}$ by 5 reduces the growth time by almost 3 orders of magnitude. In general, with falling $B_{\mathrm{d}}$ a maximum of the 
Table 3. Growth times $\tau_{\text {growth }}$ (yrs) of the PS model. For $B_{\mathrm{d}}=5 \times$ $10^{13} \mathrm{G}$ the global minimum occurs always at the age of $3 \times 10^{3} \mathrm{yrs}$.

\begin{tabular}{|c|c|c|c|c|}
\hline $\begin{array}{r}B_{\mathrm{d}} \\
(\mathrm{G})\end{array}$ & & & & \\
\hline & Profile type & Cubic & Heptic & Sinusoidal \\
\hline \multirow{4}{*}{$\begin{array}{l}\frac{n}{0} \\
x \\
n \\
n\end{array}$} & Global min. & 3500 & 2920 & 1740 \\
\hline & Global max. & 227490 & 71230 & 55330 \\
\hline & occurs at age & $10^{5}$ & $10^{5}$ & $3 \times 10^{7}$ \\
\hline & Val. at max. age & 135300 & 69630 & 55330 \\
\hline \multirow{6}{*}{$\stackrel{0}{\circ}$} & Global min. & 590900 & 68590 & 248650 \\
\hline & occurs at age & $3 \times 10^{6}$ & $3 \times 10^{3}$ & $3 \times 10^{6}$ \\
\hline & Global max. & 3071190 & 1796610 & 4540120 \\
\hline & occurs at age & $3 \times 10^{3}$ & $10^{4}$ & $10^{5}$ \\
\hline & Val. at max. age & 658290 & 342250 & 271080 \\
\hline & Gap & $10^{4} \ldots 10^{5}$ & $3 \times 10^{4} \ldots 10^{5}$ & $10^{4} \ldots 3 \times 10^{4}$ \\
\hline
\end{tabular}

Table 4. Comparison of the results for $\tau_{\text {growth }}$ of the FP and the PS models.

\begin{tabular}{lll}
\hline \hline & \multicolumn{1}{c}{ FP } & \multicolumn{1}{c}{ PS } \\
\hline $\begin{array}{l}\text { Dependence on age } \\
\text { during rapid cooling }\end{array}$ & falling & constant \\
\hline $\begin{array}{l}\text { Age at which } \tau_{\text {growth }} \\
\text { is maximum (yrs) }\end{array}$ & some $10^{6}$ & some $10^{5}$ \\
\hline $\begin{array}{l}\text { Minimum } \tau_{\text {growth }} \text { in } \\
\text { late stages }(\text { yrs })\end{array}$ & $\approx 20000$ & $\approx 55000$ \\
\hline $\begin{array}{l}\text { Sensitivity of } \tau_{\text {growth }} \\
\text { with respect to } 1 / B_{\mathrm{d}}\end{array}$ & $\begin{array}{l}\text { linear to } \\
\text { weakly nonlinear }\end{array}$ & $\begin{array}{l}\text { extremely nonlinear } \\
\text { at early ages, } \\
\text { linear from } 10^{5} \text { yrs on }\end{array}$ \\
\hline
\end{tabular}

growth time is forming between the ages of $10^{4}$ and $10^{5} \mathrm{yrs}$. "Gaps" in age without any unstable modes are listed in Table 3.

The background dynamics permissibility condition is not well satisfied at all the young stages, except for $B_{\mathrm{d}}=5 \times 10^{13} \mathrm{G}$. As the background field and diffusivity profiles don't change significantly for ages larger than $10^{6}$ yrs, the growth times are reliable from this age on. A comparison of the different profile types with respect to the later stages yields the sinusoidal one to be the most favorable for the instability, with the heptic one being close on its heels.

\subsubsection{Comparison FP - PS model}

From a final view on the results for both models we summarize the main differences between them as shown in Table 4 .

As a striking common feature of a majority of all the specific models one finds the very short growth times at the youngest ages. This is in contradiction to our assumption, introduced in Geppert \& Rheinhardt (2002) and based on the magnitude of $\omega_{B} \tau_{\mathrm{e}}$, that the Hall instability switches on only after a considerable cooling down of the NS, i.e., only after some $10^{4}$ to some $10^{5}$ yrs. An early onset of the instability could have a noticeable impact on the distribution of the magnetic field strengths across the NS population. Namely, if we had a growth time of, say, 500 yrs at the age of $3000 \mathrm{yrs}$ changing to, say, 1000 yrs at the age of 10000 yrs one would expect an essential part of the background field energy to be consumed by the unstable modes during some 10000 yrs after birth. However, we see at least two reasons to suggest a very careful use of these "early" growth times. First, during the early stages the instability may benefit from perhaps unphysically high values of the curvature parameter inherited from our initial background fields. For these, the value of the curvature parameter reached even infinity at $\rho=\rho_{\text {in }}$ because the background field profile $f(z)$ shows a kink there. For the later stages, instead, we are optimistic that this heritage lost its significance due to the long lasting period of smoothing diffusion. Second, even if the value of the curvature parameter could be regarded as realistic, one had to take account of the high sensitivity of the conductivity with respect to temperature during the hot young stage of the NS. Likewise, the high sensitivity of $\tau_{\text {growth }}$ with respect to $B_{\mathrm{d}}$ and, hence, to the curvature parameter depending on conductivity, should be considered. Thus, due to the enhanced local heating by the "hot spots" of the unstable modes (see Fig. 17) an efficient self-limitation of the mode amplitudes or even a switching-off of the instability may take place. Perhaps, this happens far before a significant part of the background field's energy is transferred to unstable modes.

\subsection{Eigenfunctions}

Figure 15 shows some typical eigenfunctions of the poloidal and toroidal scalars, $s$ and $t$, for the most favorable background field profiles, that is, the sinusoidal ones with $\rho_{\text {in }}=$ $10^{13} \mathrm{~g} \mathrm{~cm}^{-3}, B_{\mathrm{d}}=5 \times 10^{13} \mathrm{G}$ at the earliest and latest stages, respectively. Table 5 lists major qualitative differences of the eigenfunctions for the two NS models.

In general all eigenfunctions show one pronounced (primary) maximum in both $|s|$ and $|t|$ with two additional (secondary) local maxima of $|t|$ close to the vacuum boundary for the two (FP) and five (PS) last ages, respectively. The global maxima of $|s|$ lie a bit closer to the vacuum boundary than the corresponding ones of $|t|$.

With one exception, the secondary maxima are lower than the primary ones. They are sharpening with growing age and $B_{\mathrm{d}}$, and emerge immediately below and above a zero of $t$ which exists from the age of $3 \times 10^{5} \mathrm{yrs}$ on and moves towards the vacuum boundary with aging. In order to discuss this feature, we stress at first that because of its clear convergent behavior with respect to grid size it has to be considered as a physical fact rather than a numerical artifact. Since the zero of $t$ mimics a vacuum boundary (see Eq. (10)), it is intriguing to relate the peak beneath it to the similar peak right beneath the vacuum boundary in the homogeneous density model (see Rheinhardt \& Geppert 2002).

Obviously, the perturbation fields concentrate mainly in regions of high values of $B_{0}$ and/or the curvature parameter, respectively.

To judge whether the field structures of the eigenmodes might be considered small-scale, we state first that their 

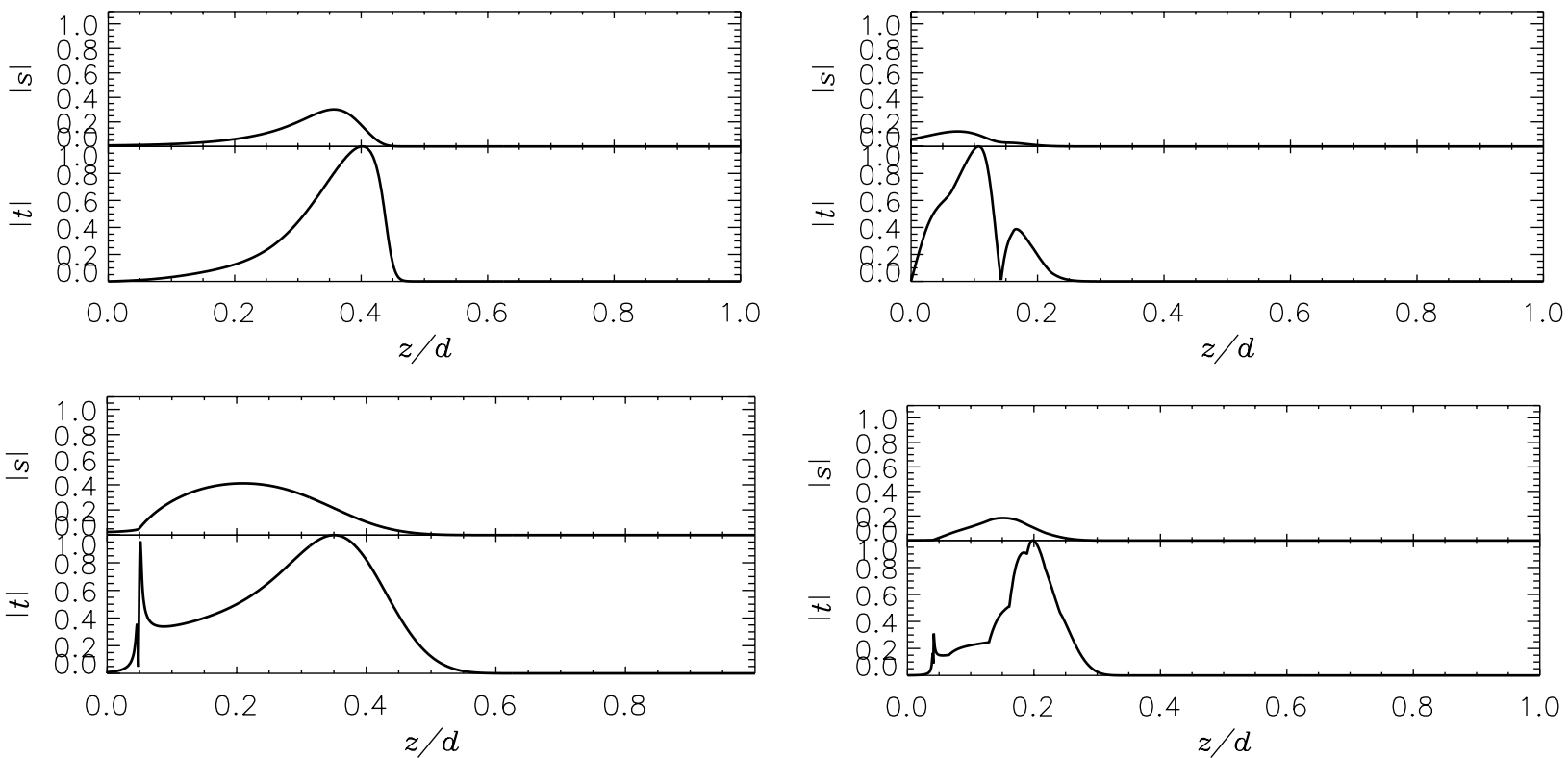

Fig. 15. Moduli of the scalars $s$ and $t$ of the fastest growing mode for the FP (left) and PS (right) model with the sinusoidal initial background field profile Eq. (15), initial field $B_{\mathrm{d}}=5 \times 10^{13} \mathrm{G}$, initial penetration density $\rho_{\text {in }}=10^{13} \mathrm{~g} \mathrm{~cm}^{-3}$ at the ages of $3 \times 10^{3}$ yrs (above) and $3 \times 10^{7}$ yrs (below).

Table 5. Comparison of qualitative features of the eigenfunctions (cf. Figs. 15 and 16).

\begin{tabular}{lll}
\hline \hline & \multicolumn{1}{c}{ FP model } & \multicolumn{1}{c}{ PS model } \\
\hline $\begin{array}{l}\text { Primary maximum } \\
\text { of }|s| \text { and }|t|\end{array}$ & $\begin{array}{l}\text { almost fixed near } z / d=0.4 \text { except at last age } \\
\text { when closer to surface: } \\
\text { at } z / d=0.2 \text { for }|s| \text { and } z / d=0.35 \text { for }|t|\end{array}$ & almost fixed near $z / d=0.2$ \\
\hline Secondary maxima of $|t|$ & $\begin{array}{l}\text { lower than corresponding primary maximum } \\
\text { except at last age }\end{array}$ & $\begin{array}{l}\text { always lower than primary maximum } \\
\text { "early" secondary maximum at } z / d=0.1 \text { at } \\
\text { age } 3 \times 10^{3} \text { yrs }\end{array}$ \\
\hline Inner zero of $|t|$ & $\begin{array}{l}\text { emerges at age } 3 \times 10^{6} \text { yrs, moves from } \\
z / d=0.2 \text { to } z / d=0.05 \text { with growing age }\end{array}$ & $\begin{array}{l}z / d=0.07 \text { to } z / d=0.04 \text { with growing age; } 3 \times 10^{5} \text { yrs, moves from }=0.14 \text { at age } 3 \times 10^{3} \text { yrs. } \\
\text { "early" zero at } z / d=0.14\end{array}$ \\
\hline
\end{tabular}

major radial scale corresponds with the major radial scale of the corresponding background field. The tangential scales can simply be identified with (half of the) tangential period lengths $\lambda$, which again reflect the radial background field scale. When comparing with the crust thickness, there are surely no small scales, except the structures around the secondary maxima. But, when comparing with the NS perimeter it is well justified to claim small-scaleness since for the FP model the $\lambda^{\max }$ lie between 700 and $1000 \mathrm{~m}$, whereas the NS's perimeter is $67 \mathrm{~km}$. For the PS model we have $\lambda^{\max }=1500 \ldots 4500 \mathrm{~m}$ with a perimeter of $103 \mathrm{~km}$.

Because the non-smoothness of the conductivity profiles in the PS model is not directly expressing itself in the background field profiles, one could expect the same for the eigenfunctions. Indeed, the eigenfunctions of the first four ages don't show such signatures, but those of the five last ages do. This fact is only partly explainable by the sharper kinks in the $\eta$ profiles of the last four ages in comparison with those of the first five (see Fig. 4). It strengthens again the impression that there is a qualitative difference between "earlier" and "later" stages of the PS model.

Figure 16 shows perturbation field structures for selected cases. As a general tendency the fields of the FP model reach deeper layers of the crust and are less concentrated in $z$-direction than those of the PS model. At early stages the fields tend to be relatively stronger above the slab in comparison with their maxima inside. Note the very sharp maxima of $b_{y}$ near the surface for the latest stages of the FP model, which are connected with the occurrence of secondary maxima in the toroidal scalar $t$ (see Fig. 15).

\section{Summary and conclusions}

The results presented here prove that the instability, originally found in a slab of uniform density, does persist when a typical stratification of density and chemical composition as present in NS crusts, and a realistic background field are employed. 

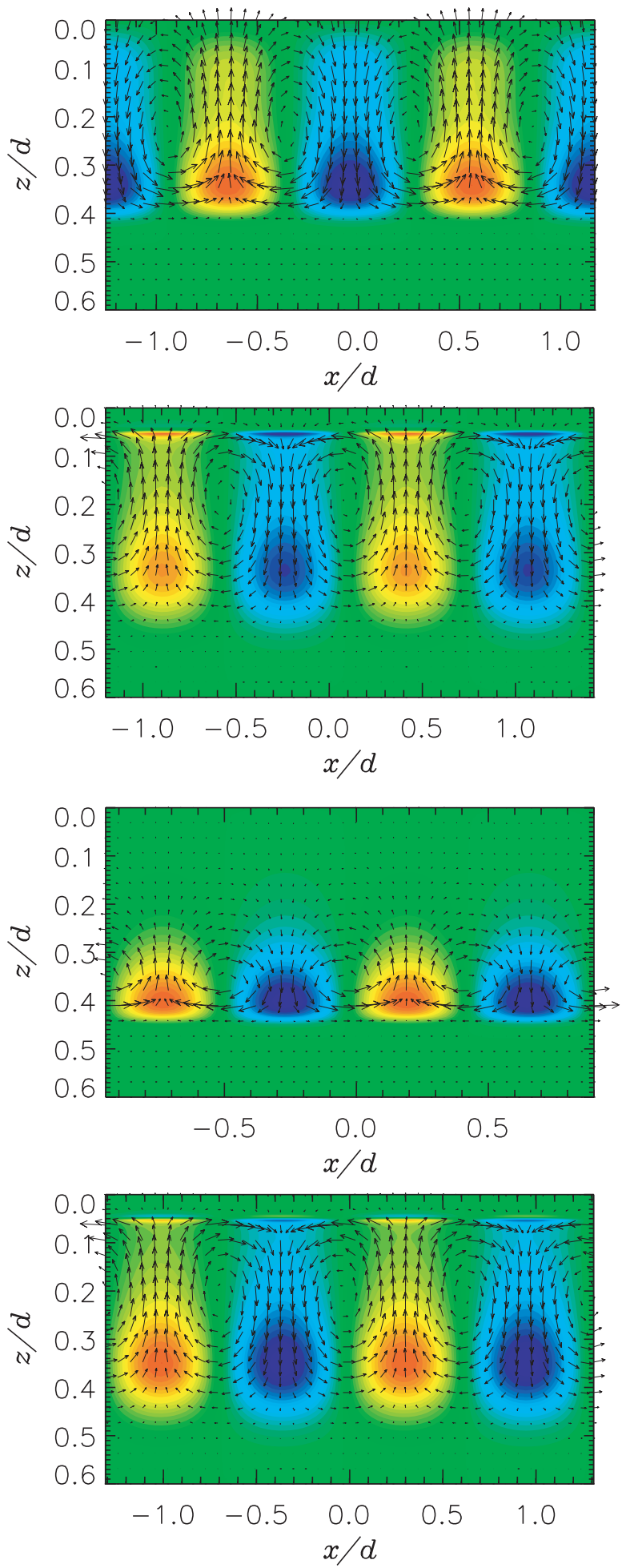

$3 \times 10^{3}$
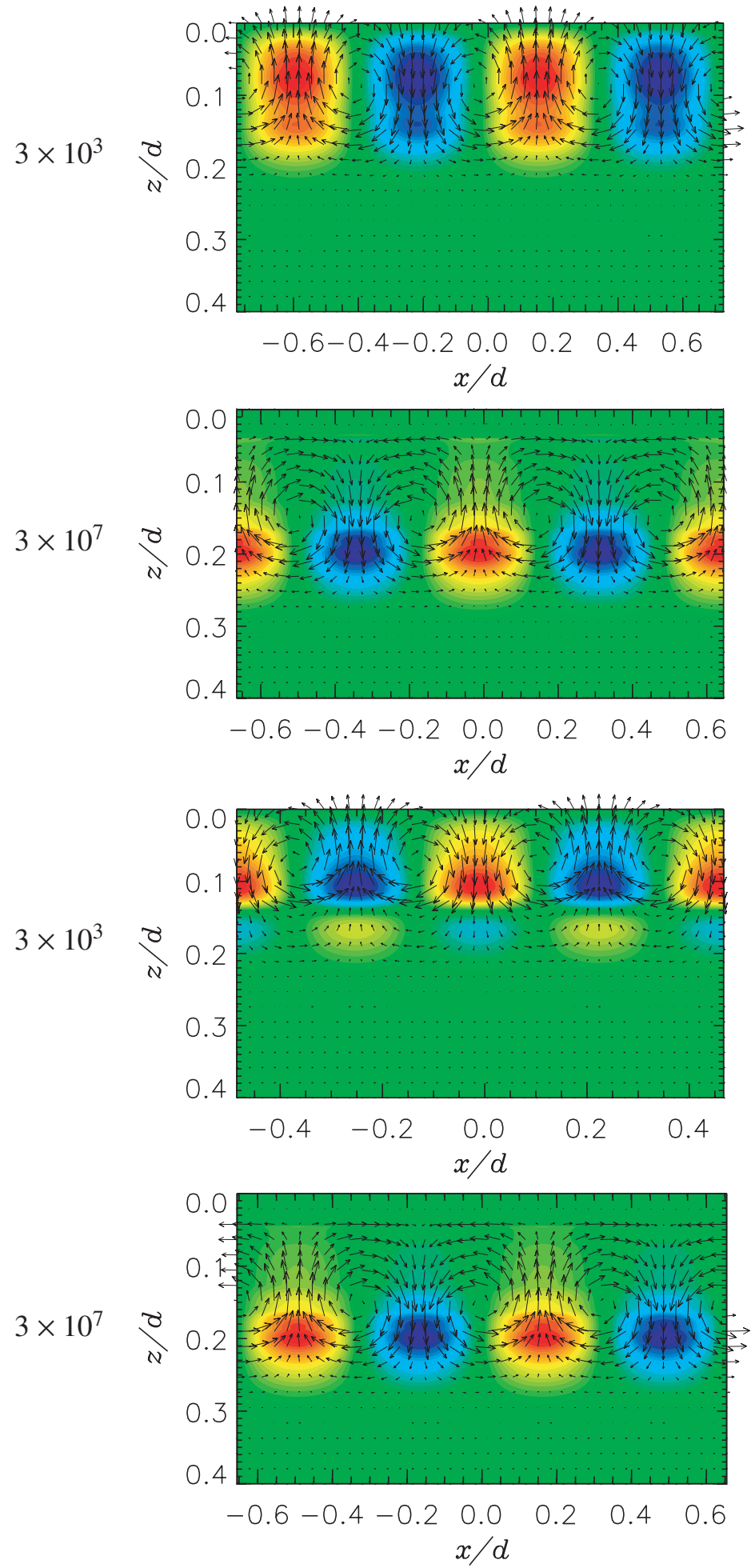

Fig. 16. Fields of the fastest growing modes for the FP (left $)$ and PS (right) model, initial field $B_{\mathrm{d}}=5 \times 10^{13} \mathrm{G}$, initial penetration density $\rho_{\text {in }}=$ $10^{13} \mathrm{~g} \mathrm{~cm}^{-3}$. Upper two rows: cubic Eq. (13), lower two rows: sinusoidal Eq. (15) initial background field profile. Ages as indicated in the middle (in yrs). Perpendicular field component: light (in color: green to red) - into, dark (in color: green to blue) - out of the paper plane.

We found that independent of the specific NS model, the strength and structure of the background field, the growth times are smallest at early stages of the NS, reaching then a maximum at a model depending intermediate age and become smaller again in the process of further cooling. In some cases (e.g.,
PS model, $B_{\mathrm{d}}=10^{13} \mathrm{G}$ ) the instability disappears for too low background field strengths in a period of intermediate ages.

In order to understand this behavior, note that during the early period the crustal crystal is so hot that a large number of phonons is excited. Hence, the electron relaxation time $\tau_{\mathrm{e}}$ is 
relatively small, causing in turn a small magnetization parameter $\omega_{B} \tau_{\mathrm{e}}$. But, competing with that, the magnetic field, initially confined to a shallow crustal layer had not yet time enough neither to decay nor to diffuse deeper into the crust therefore still preserving the curvature of its initial profile. It seems, that this effect dominates the relative smallness of $\omega_{B} \tau_{\mathrm{e}}$. In turn, during late, cool stages $\tau_{\mathrm{e}}$ is much larger but the curvature of the background field profile is due to its progressing diffusion and decay considerably smaller. (See Figs. 5 to 7.)

Quite general, our conclusion in Rheinhardt \& Geppert (2002) that the appearance of the instability depends both on a sufficiently strong curvature of the background field profile and a sufficient large magnetization parameter $\omega_{B} \tau_{\mathrm{e}}$ has thus been confirmed. However, it turned out that in models with stratification one has to include the derivative of the Hall coefficient into the notion of "curvature" by introducing a properly defined curvature parameter (see Sect. 2). It estimates the ratio of the most relevant terms in the governing Eqs. (3).

The differences between growth times within the set of early stages (instants labeled 1 to 5 in Figs. 5 to 7) or late stages (instants labeled 7 to 9), respectively, are well reflected by the curvature parameter. But, unfortunately, it fails when comparing growth times between the two sets as it predicts the shortest growth times for the late stages. In both NS models studied, there seem to exist subtle and hidden features of the coefficients and background field profiles which cause a qualitative contrast between early and late stages not understood up to now.

Our results give rise to the idea that the Hall instability will act relatively early in the NS's life. Hence, it could reduce and smooth out the background field in its progress, so that later on (say after $10^{5} \mathrm{yrs}$ ) the conditions for the occurrence of the instability are no longer given.

On the other hand we found (mainly for the PS model) a high sensitivity of the "early" growth times with respect to the background field strength. An increase by a factor of 5 can cause a decrease of the growth time by almost three orders of magnitude. Recalling the importance of the profile curvature, we conclude that details of the background field (strength and structure) determined by the generation process of the NS magnetic field may play a crucial role with respect to appearance and vigour of the instability at early stages. Since these processes are far from being completely understood up to now, the only detail one can discuss is the initial radial extent of the generated fields. Generation by a thermoelectric instability in the relatively thin liquid shell forming the later crust surely provides radial profiles with a lot of curvature. But whether they are really instability-friendly has to be put in question as a small initial penetration depth of the background field $\left(\rho_{\text {in }}=10^{12} \mathrm{~g} \mathrm{~cm}^{-3}\right)$ turned out to be non-favorable. Considering alternatively typical structures of convection in a nascent NS (see, e.g., Keil et al. 1996; Fryer \& Heger 2000) one might conclude, that the assumption of bigger initial penetration depths could be appropriate for magnetic fields generated by a proto-NS dynamo. In all, we suggest to make use of the "early" growth times with great care only.

The late stages, in contrast, are increasingly less influenced by peculiarities of the somewhat arbitrary assumptions on the initial fields. At these stages the dependence of the growth times on the background field strength is undramatic (close to inversely linear). So we think that we can rely safely on the growth times obtained at ages $\gtrsim 10^{5}$ yrs.

If the Hall instability sets on at late stages, small-scale perturbations amplified at the expense of the background field may survive for a long time. To estimate it, let us first look onto the Ohmic decay times for typical perturbations. Their scale lengths are in the order of $5 \times 10^{4} \ldots 10^{5} \mathrm{~cm}$, and their field maintaining currents circulate in a depth of $2 \ldots 5 \times 10^{4} \mathrm{~cm}$ corresponding to a density of about $10^{12} \mathrm{~g} \mathrm{~cm}^{-3}$. Taking into account that at an age of $10^{5} \ldots 10^{6}$ years the conductivity in that region is in the order of $10^{26} \ldots 10^{27} \mathrm{~s}^{-1}$, we arrive at values of $10^{7} \ldots 10^{8}$ years. However, these perturbations together with the background field are subject to the Hall effect. Following a usual argumentation, one would therefore expect that the real decay times are shorter than the ohmic ones. This conclusion would be based on the idea, that the Hall effect, although being conservative on its own, leads to an accelerated decay of a large scale field because energy is flowing along the Hall cascade towards small scales the dissipation rate of which is higher than that of the large scale field. However, this is not the whole truth for a situation in which energy is already concentrated in a small scale. Along with a Hall cascade to even smaller scales dissipating more quickly another one to larger scales can occur which dissipate more slowly. Whether or not such a double-sided cascade leads to an overall acceleration of the decay of the original small-scale mode must remain open.

The possible occurrence of relatively persistent small-scale field structures at the NS surface together with the (episodically) accelerated decay of the large-scale background field caused by the energy transfer to small-scale modes during their rapid growth represent the most important aspects of the Hall instability with respect to observations. Possible observable consequences are therefore

- deceleration of the NS spin-down due to the accelerated magnetic field decay. Note, that the real extent and duration of the latter can only be determined by analyzing the nonlinear stage of the instability including saturation;

- a hotter NS surface as a consequence of enhanced Joule heating due to the concentration of magnetic energy in the small scales of unstable eigenmodes;

- glitches and bursts as a consequence of enhanced Lorentz forces making crust cracks more probable;

- emergence of radio subpulses due to the existence of smallscale structures in the vicinity of the magnetic pole.

A discussion of the first three effects can be found in Geppert \& Rheinhardt (2002). We will not repeat it here since all the general arguments hold true for the Hall instability in a stratified crust, too. However, some modification seems to be necessary with respect to the enhancement of Joule heating, since in our present results the case of "hot spots" close to the surface is not the prevailing one (Fig. 17). Thus, the argument, that small-scale temperature features due to localized Joule heating will be washed out and remain unobservable in the light curve seems to be the more valid. On the other hand, an observable rise in the average surface temperature in comparison with the standard cooling due to the additional "deep" Joule heating is 


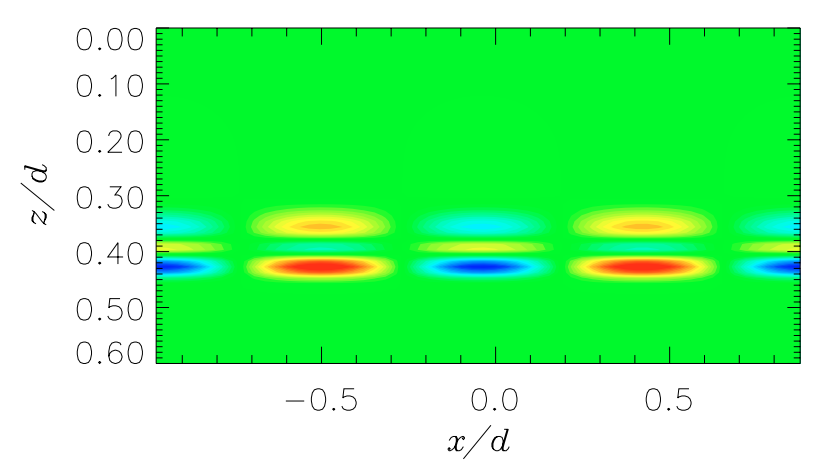

Fig. 17. Perturbation of the Joule heat sources density $(\eta / 2 \pi) \operatorname{curl} \boldsymbol{B}_{0}$. curlb in arbitrary units corresponding to the perturbation field of the left column, third row of Fig. 16. Light (in color: green to red) - positive, dark (in color: green to blue) - negative deviations from the background heat sources.

likely at least if the instability acts during the phase for which the standard model predicts a rapid cooling $\left(10^{5} \ldots 10^{6} \mathrm{yrs}\right)$.

A necessary ingredient of the pulsar vacuum gap model of Ruderman \& Sutherland (1975) is the existence of relatively strong and small-scaled poloidal magnetic field structures just above the surface of the polar cap. In Geppert et al. (2003) we demonstrated that the characteristics of such a field as derived by Gil et al. (2003) can be found with some of the unstable modes presented here.

Acknowledgements. M.R. and U.G. gratefully acknowledge financial support by the Arbeitsamt Berlin and the hospitality of the AIP. D.K. is thankful to the Humboldt foundation for the fellowship realized with the hospitality of the AIP and to the support of grant Nr. 03-02-17522 of RFBR.

\section{References}

Baym, G., Pethick, C., \& Sutherland, P. 1971, ApJ, 170, 299

Becker, W., Swartz, D. A., Pavlov, G. G., et al. 2003, ApJ, 594, 798

Friedman, B., \& Pandharipande, V. R. 1981, Nucl. Phys. A, 361, 302

Fryer, C. L., \& Heger, A. 2000, ApJ, 541, 1033

Geppert, U., \& Rheinhardt, M. 2002, A\&A, 392, 1015

Geppert, U., Rheinhardt, M., \& Gil, J. 2003, A\&A, 412, L33

Gil, J., Melikidze, G., \& Geppert, U. 2003, A\&A, 407, 315

Gil, J. A., Melikidze, G. I., \& Mitra, D. 2002, A\&A, 388, 235

Goldreich, P., \& Reisenegger, A. 1992, ApJ, 395, 250

Haensel, P., Urpin, V., \& Yakovlev, D. 1990, A\&A, 229, 133

Haensel, P., \& Zdunik, J. L. 1990, A\&A, 229, 117

Hollerbach, R., \& Rüdiger, G. 2002, MNRAS, 337, 216

Johnston, S., \& Galloway, D. 1999, MNRAS, 306, L50

Keil, W., Janka, H.-T., \& Müller, E. 1996, ApJ, 473, L111

Misner, C. W., Thorne, K. S., \& Wheeler, J. A. 1973, Gravitation (San Francisco: Freeman)

Naito, T., \& Kojima, Y. 1994, MNRAS, 266, 597

Negele, J. W., \& Vautherin, D. 1973, Nucl. Phys. A, 207, 298

Page, D., Geppert, U., \& Zannias, T. 2000, A\&A, 360, 1052

Pandharipande, V. R., \& Smith, R. A. 1975, Nucl. Phys. A, 237, 507

Rheinhardt, M., \& Geppert, U. 2002, Phys. Rev. Lett., 88, 101103

Ruderman, M., \& Sutherland, P. 1975, ApJ, 196, 51

Shalybkov, D. A., \& Urpin, V. 1995, MNRAS, 273, 643

Shalybkov, D. A., \& Urpin, V. 1997, A\&A, 321, 685

Thompson, C., \& Duncan, R. 1993, ApJ, 408, 194

Urpin, V., Chanmugam, G., \& Sang, Y. 1994, ApJ, 433, 780

Urpin, V., \& Konenkov, D. 1997, MNRAS, 292, 167

Urpin, V., \& Shalybkov, D. 1995, MNRAS, 294, 117

Urpin, V., \& Shalybkov, D. 1999, MNRAS, 304, 451

Urpin, V., \& Yakovlev, D. 1980, Sov. Astron., 24, 425

Vainshtein, S., Chitre, S., \& Olinto, A. 2000, Phys. Rev. E, 61, 4422

van Riper, K. 1991, ApJS, 75, 449

Wiebicke, H.-J., \& Geppert, U. 1996, A\&A, 309, 203

Yakovlev, D., \& Shalybkov, D. 1991, Ap\&SS, 176, 171 